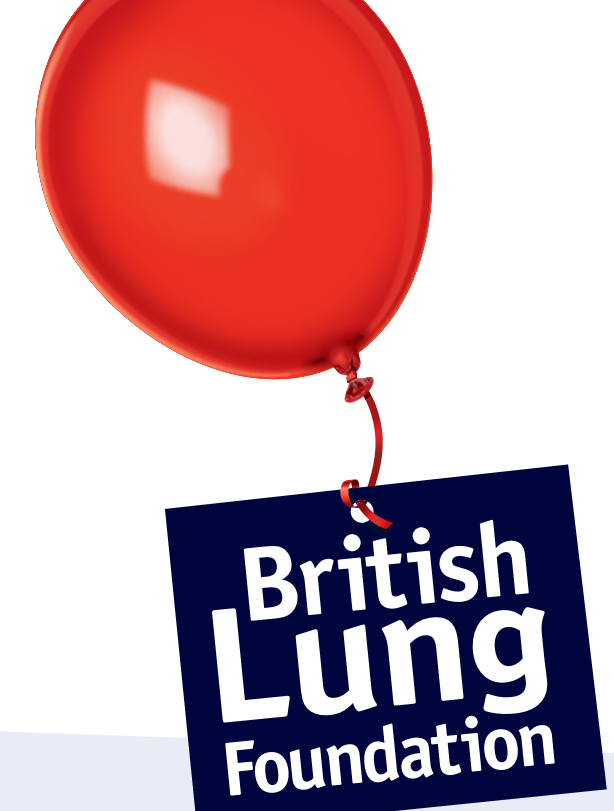

\title{
Obstructive Sleep Apnoea (OSA)
}

Toolkit for commissioning and planning local NHS services in the UK 2015 


\section{Contents}

Forewords

1 Executive Summary $\quad 4$

2 Introduction $\quad 5$

3 About OSA 6

3.1 The burden of OSA 6

3.2 The risk of OSA across the UK 9

$\begin{array}{ll}\text { 3.3 The cost of OSA - health economics } & 10\end{array}$

4 Service provision and planning 12

4.1 The patient pathway - recommended OSA service provision $\quad 12$

4.2 Planning and commissioning - recommended process $\quad 21$

4.3 Audit and calculator tools 23

5 Appendix $\quad 29$

\begin{tabular}{ll}
5.1 Referral protocol example & 29 \\
\hline
\end{tabular}

5.2 Key performance indicators - outputs and outcomes 31

5.3 Patient survey example 32

$\begin{array}{ll}5.4 \text { Endorsements and acknowledgements } & 40\end{array}$

5.5 Data sources and methodology used to develop OSA risk map and calculator $\quad 42$

To find out more about OSA and our campaign, visit www.blf.org.uk/OSA 


\section{Emeritus Professor John Stradling}

My interest in sleep medicine, and OSA in particular, developed many years ago as a result of seeing patients who clearly had a major problem, but nobody knew what it was.

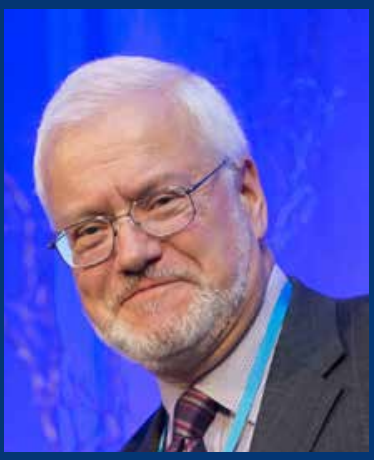
I have seen thousands of patients over the years, and providing them with effective CPAP therapy has been one of the most rewarding aspects of my career; it literally changes lives. People come into clinic feeling like giving up, feeling that they are living in a fog, and many saying that they are at risk of falling asleep at the wheel. They then leave being able to return to their daily lives, with a renewed energy and outlook on life. This is because they have regained the ability to sleep properly, something many of us take for granted. Yet despite the massive difference that treatment makes to people with OSA and their families, OSA is still under-recognised, under-diagnosed, and thus undertreated, leaving many people at risk of other health complications as a result. This means of course that they remain a major burden on the NHS. In addition, it places people at increased risk of causing road collisions and therefore is a burden on society as a whole.

Where sleep clinics do exist, they are usually struggling to keep up with increasing referrals, and thus the increasing demand to provide ongoing support and management to patients after treatment has started. The time has come to address these issues.

This OSA toolkit brings together for the first time and in a readable format, all the key findings and guidelines on OSA and offers practical guidance on how to set up and run a sleep service. I hope that it will be used by commissioners and service planners to set up and deliver services that are both efficient and effective. This is a rare opportunity for proactive purchasing which will genuinely lead to a reduction in future health costs across a range of NHS services.

\section{Dr Penny Woods, Chief Executive, British Lung Foundation (BLF)}

I know from personal experience how much OSA affects family life, and one of the reasons I joined the BLF was

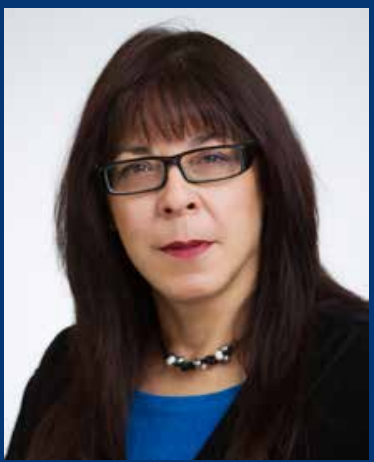

because I was impressed by the campaigning work being done to raise awareness of OSA and improve service provision. We have been leading on this campaign since 2011 and the launch of this toolkit marks the culmination of the years we have spent listening to patients, health care professionals and commissioners. The toolkit references, uses and builds on our previous work: we mapped OSA risk and predicted prevalence against sleep service provision across the UK, and, for the first time, identified a mismatch between service provision and potential need for services; we conducted the largest ever survey of people with OSA in the UK; we hosted the first UK health care professional conference on OSA and we commissioned a UK OSA health economics report.

We are proud of the enormous progress that has been achieved. But we also know there is still a long way to go in order to ensure that people with OSA get the right diagnosis and are offered the right treatment, wherever they live in the UK. We hope that this toolkit will be a useful resource for health care professionals, service planners and commissioners to help plan services to meet the needs of local populations of people with OSA and their families. 


\section{Executive summary}

Obstructive Sleep Apnoea (OSA) is a sleeprelated respiratory condition, leading to repeated temporary cessations of breathing because of a narrowing or closure of the upper airway during sleep. Core symptoms of OSA include excessive daytime sleepiness, snoring, and witnessed apnoeas or hypopnoeas (blockages or partial blockages of the airway leading to breathing cessations).

OSA can affect anyone, but is more common in some people, e.g. those who are male, middle aged, elderly, and over-weight.

OSA is common, affecting an estimated 1.5 million adults in the UK, and yet up to $85 \%$ are undiagnosed, therefore untreated.

Undiagnosed OSA is closely associated with serious health problems, including hypertension, diabetes, stroke and heart disease. It can shorten life expectancy and it also leads to road collisions caused by undiagnosed sleepy drivers. In short, OSA is currently a significant health care burden in the UK, for individuals, for the NHS, and for society as a whole. Although awareness of OSA is rising in the population, $42 \%$ of people who snore or whose partner snores have not even heard of OSA. GP awareness levels are also low.

Service provision across the UK varies hugely, and some areas with a high risk of predicted OSA prevalence have lower service provision levels than other areas where there is more provision and less risk. OSA is relatively easy to treat, and its treatment has been proven to be cost effective. For many years, research has proven that treatment is associated with better outcomes for the individual and for society. And yet, only an estimated 330,000 adults are currently being treated, out of an OSA population of 1.5 million.

Treating OSA with continuous positive airway pressure (CPAP) offers greater value for money than many other NICE recommended technologies.

Treating OSA would save the NHS millions of pounds.
Estimated annual savings to the NHS in the UK would total $£ 55$ million and 40,000 QALYs, if all people with moderate to severe OSA were to be diagnosed and treated, relative to none being diagnosed and treated.

The estimated annual savings to the NHS in the UK would be $£ 28$ million and 20,000 extra QALYs, if all people with moderate to severe OSA were to be diagnosed and treated, compared with the current estimated level of treatment of 330,000 adults.

This toolkit has been developed by the BLF to support anyone involved in commissioning and planning services for OSA in the UK. It is designed to help audit existing services, understand local needs and plan future provision. The toolkit accommodates the different commissioning and funding models across the UK.

The toolkit consists of this booklet and an online OSA calculator tool. The booklet contains recommendations for service provision and commissioning and includes an audit checklist. The online tool generates an OSA report for a particular health "area" (Clinical Commissioning Groups (CCGs) in England, Health and Social Care Trusts (HSCTs) in Northern Ireland, NHS Health Boards (HBs) in Scotland and Local Health Boards (LHBs) in Wales).

The report includes relevant information about the local population, details about cost avoidance for treating versus not treating OSA, and advice on staffing levels.

The toolkit has been developed using existing UK guidelines and findings from the BLF's OSA project. 


\section{Introduction}

\section{5 key reasons why OSA is important}

1. OSA is common and under-diagnosed

2. OSA is closely linked to other serious health problems

3. Awareness levels are low

4. Service levels vary across the UK

5. Treatment is straightforward and cost effective, and would save the NHS millions

\section{About the toolkit}

\section{What is it for?}

This toolkit has been developed by the BLF to support anyone involved in commissioning and planning services for OSA in the UK. It is designed to help audit existing services, understand local needs and plan future provision. The toolkit takes into consideration that there are different commissioning and funding models across the UK.

\section{How to use the toolkit}

The easy to use resource consists of this booklet, and an accompanying web-based OSA service calculator tool. To make full use of the toolkit you will need to:

\section{Read this booklet}

2. Complete the audit checklist in this booklet about current OSA service provision that you offer

3. Go online to our OSA service calculator tool, enter your health area name (Clinical Commissioning Groups (CCGs) in England, Health and Social Care Trusts (HSCTs) in Northern Ireland, NHS Health Boards (HBs) in Scotland and Local Health Boards (LHBs) in Wales), and download your OSA report. You can download as many reports as you like

4. Share the audit checklist and OSA report with key stakeholders in your area to help you with service planning and commissioning
The booklet describes the burden of OSA in the UK, explains OSA risk across the UK, and highlights the geographical differences in service provision. The economic case for treating OSA is provided. This is followed by recommendations for service provision for a "typical" area, based on existing guidelines and research.

The audit checklist enables health areas to benchmark themselves against recommended provision. Links to relevant websites and documents are provided. There is also a link to the web-based OSA service calculator tool.

The OSA service calculator tool generates a report for a named local health "area" (Clinical Commissioning Groups (CCGs) in England, Health and Social Care Trusts (HSCTs) in Northern Ireland, NHS Health Boards (HBs) in Scotland and Local Health Boards (LHBs) in Wales).

The report provides information on the adult local population, the estimated OSA prevalence, recommended service provision for a population of this size, cost avoidance for treating OSA, and information about the local prevalence of OSA risk factors to consider when making decisions about service provision.

Finally, we would like to work with you to find out whether this toolkit has been useful. We ask you to complete two evaluation questions online once you have read your report. Also, if you give us your contact details when you access your report online, we can keep in touch. 


\section{About OSA}

\subsection{The burden of OSA}

Obstructive Sleep Apnoea (OSA) is a sleep-related respiratory condition, leading to repeated temporary cessations of breathing because of a narrowing or closure of the upper airway during sleep. OSA, when untreated, deprives people of a healthy sleep, which can cause severe daytime sleepiness.

Figure one:

The cycle of OSA during sleep

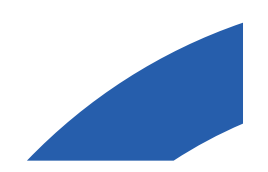

Airway in the throat closes or partially closes (obstructs)

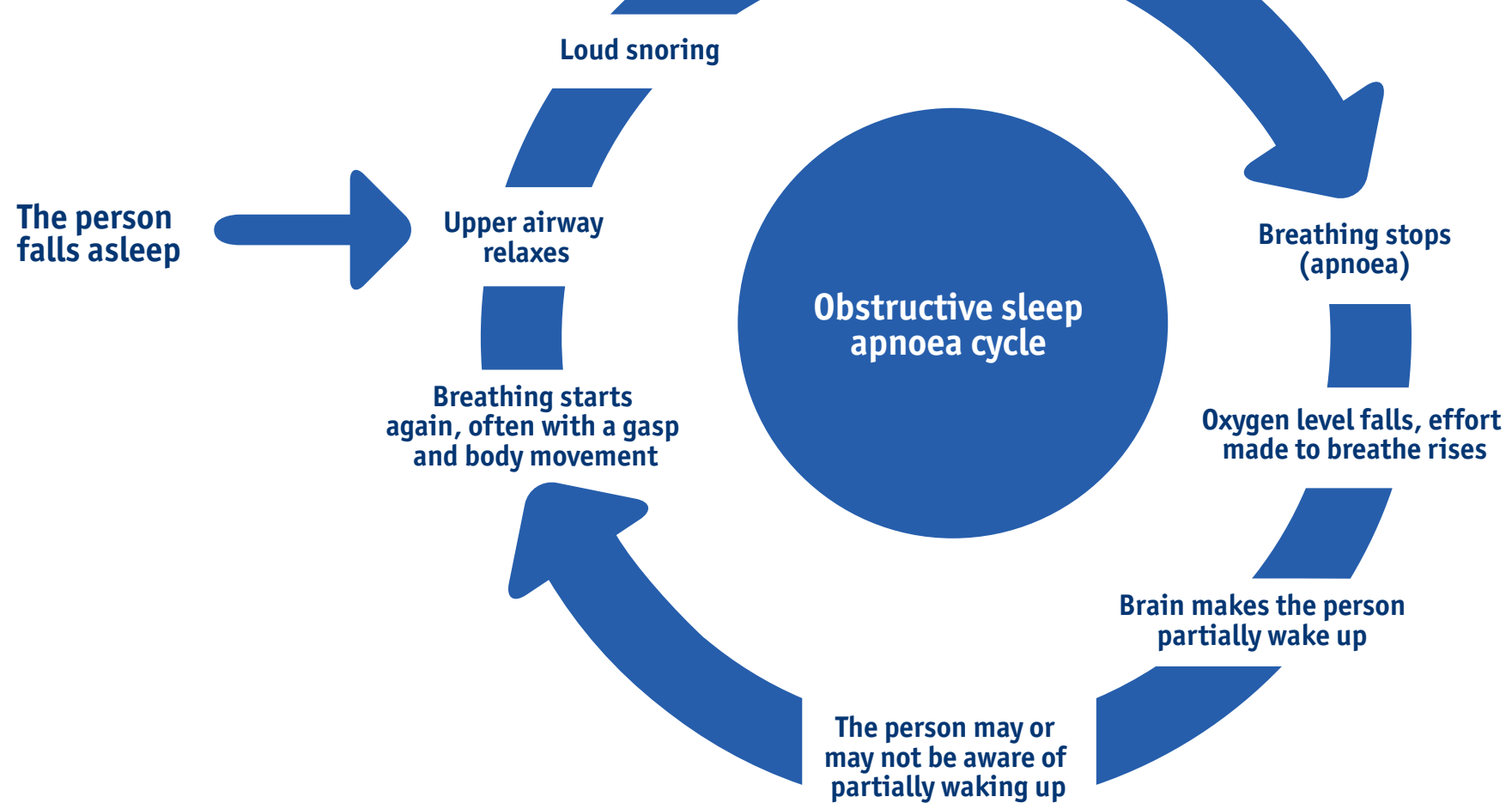

Core symptoms of OSA include excessive daytime sleepiness, snoring, and witnessed apnoeas or hypopnoeas (blockages or partial blockages of the airway leading to breathing cessations).

Health care professionals and the literature sometimes refer to OSAS (obstructive sleep apnoea syndrome), which is OSA combined with symptoms, the most common of which is sleepiness. Also used is the term OSAHS (obstructive sleep apnoea hypopnoea syndrome).
For the purposes of this document, we shall use the term OSA but we use this term to include people with symptoms.

OSA can affect anyone, but is more common in some people, including those who:

- snore

- intermittently stop breathing when sleeping

- are male and middle aged 
- are a woman past menopause

- are overweight or obese

- have a large neck size (17 inches or more)

- have a small airway

- have a set-back or small lower jaw

- have large tonsils

- have a large tongue

- have an abnormal face shape, or nasal blockage

- have a medical condition that makes some of these factors more likely, such as Down's syndrome.'

OSA is a major health challenge in the UK. The number of adults with OSA in the UK who are being treated is around 330,000 , out of a total population of adults with OSA of around 1.5 million. ${ }^{2}$

Yet recent evidence suggests that $85 \%$ of people with OSA in the UK are undiagnosed and therefore untreated. ${ }^{3}$

OSA can have a significant impact upon those it affects, not just for patients, but also for their family and colleagues. It affects people's health and wellbeing, their quality of life, and their ability to work.

Undiagnosed OSA increases the risk of road accidents, including fatal collisions.

Risk factors commonly associated with OSA are: gender (more common in men than women), age (more common in older age), hypertension, diabetes, and obesity. ${ }^{4}$ It is likely that the prevalence of OSA will rise in the coming years, particularly due to an increasing prevalence of obesity and the increasing age of the UK population.
"It felt like I was semi-comatose, living in a fog."

"I woke up feeling exhausted. I could not concentrate at work, I was depressed."

\section{"I sometimes even dozed off at the wheel."}

\section{Weight}

People who are very overweight are more at risk of developing OSA than those not overweight. Estimates vary, but around half of people with OSA have excess body weight. Approximately $40 \%$ people who are obese have OSA, while $77 \%$ of people who are morbidly obese have OSA. ${ }^{5}$

\section{Hypertension and cardiovascular disease}

There is strong evidence that OSA is a significant risk factor for cardiovascular disease and mortality. OSA prevalence is higher among patients with cardiovascular conditions. Research has shown that people with OSA have higher blood pressure, and untreated OSA is associated with a risk of arrhythmias, stroke and coronary heart disease. Death rates from cardiovascular disease are high in people with OSA, the rate increasing with the severity of the OSA. In one large study, cardiovascular disease accounted for $42 \%$ of deaths in people with OSA, compared to $26 \%$ of people without the condition. ${ }^{6}$ Between $30 \%$ and $57 \%$ of patients with coronary artery disease have also been found to have OSA.?

${ }^{5}$ O'Keeffe T, Patterson E. 2004: Evidence supporting routine polysomnography before bariatric surgery. Obes Surg. 2004;14(1): 23-6 http://www.iuns.org/features/ obesity/obesity.htm

${ }^{6}$ Fin Young et al. 2008: Sleep disordered breathing and mortality: eighteen-year follow-up of the Wisconsin sleep cohort. Sleep; 31(8), pp. 1071-8

${ }^{7}$ Won Lee MD et al. 2008: Epidemiology of obstructive sleep apnea: a population-based perspective. Expert review of respiratory medicine. June 1; 2(3), pp. 349-364
} 


\section{Diabetes}

OSA is an independent risk factor for diabetes, as it is associated with changes in glucose metabolism which places patients at increased risk of development of type 2 diabetes. ${ }^{8}$ At least $70 \%$ of people living with type 2 diabetes have some form of sleep disordered breathing. ${ }^{9}$ Studies have found that the percentage of people living with diabetes who also have OSA to be anywhere between $17^{10}$ and $48 \% .^{11}$

Evidence also suggests a relationship between OSA, obesity and diabetes: $86 \%$ of obese type 2 diabetes patients have OSA.

\section{Age}

Older people are more at risk, with 15 to $20 \%$ of those aged 70 and over estimated to have the condition. ${ }^{2}$

\section{Awareness of OSA}

Awareness levels of OSA in the UK are low.

BLF surveys conducted in 2011 and 2014 indicate that although awareness of OSA is rising in the population, with figures in 2014 suggesting that $58 \%$ of people who snore or whose partner snores have heard of OSA, this still means that $42 \%$ of people have not even heard of OSA. ${ }^{13}$

Awareness levels amongst GPs are low. In the BLF patient survey, ${ }_{1}^{14} 85 \%$ of people later diagnosed with OSA went to their GP about their symptoms. Nearly $11 \%$ of people were told not to worry or were given advice and no action was taken. Over $20 \%$ of people visited their GP on three or more occasions with their symptoms, and $7.5 \%$ of people visited the GP on five or more occasions.

\section{OSA and driving}

People whose work involves driving are particularly at risk if they have undiagnosed OSA, because if they fall asleep at the wheel they may, and unfortunately DO, cause collisions. The current estimate for the prevalence of OSA in HGV drivers is over $15 \% .^{15}$

People in other vigilance-critical roles are also affected; untreated OSA affects people's ability to concentrate due to excessive sleepiness, and increases the risk of accidents at work.

In the BLF's survey of people with OSA, at the time of diagnosis $22 \%$ had been doing a job that required them to drive regularly. $11 \%$ of people who drove had fallen asleep driving, and just fewer than two\% admitted to having a road traffic accident caused by sleepiness. $27 \%$ of these people were professional drivers. ${ }^{16}$

OSA is therefore not just a risk for the individual, but also to society as a whole due to the increased risk of fatal accidents. The impact of these road collisions, which include fatalities and lifelong disability, are associated with great emotional distress and broader societal costs. ${ }^{17}$ One fatal accident is estimated to cost approximately $£ 1.5$ million to society, ${ }^{18}$ and sleepiness accounts for around $20 \%$ of all road collisions. Many of these are likely to be caused by people with undiagnosed OSA although exact figures are unavailable.

\footnotetext{
${ }^{8}$ Caples SM, et al. 2005: Obstructive sleep apnea. Annals of Internal Medicine 142 (3), pp.187-97

${ }^{9}$ Einhorn et al 2007: Prevalence of sleep apnea in a population of adults with type 2 diabetes mellitus, Endocr Pract

${ }^{10}$ West et al 2006: Prevalence of obstructive sleep apnoea in men with type 2 diabetes, Thorax 61, 945-950

"Einhorn et al 2009: Prevalence of sleep apnea in a population of adults with type 2 diabetes mellitus, Endocr Pract, 2007; Foster et al, Diabetes Care

${ }^{12}$ Martinez-Garcia MA et al. 2010: Sleep apnoea in patients of elderly: care activity in Spain (2002-2008). Archivos de Bronconeumologia. 46 (10): $502-507$

${ }^{13}$ BLF 2014: online OSA awareness survey

${ }^{14}$ BLF 2014: OSA patient experience survey

${ }^{15}$ OSA partnership 2014: Four week wait campaign for the treatment of obstructive sleep apnoea syndrome (OSAS) for vocational drivers

${ }^{16}$ BLF 2013: Having OSA in the UK: what the patients say

${ }^{17}$ BTS 2014: Position statement on driving and obstructive sleep apnoea (OSA)

${ }^{18}$ Mackay, T. 2010: OSA working towards the development of minimal standards for referral, investigation and treatment in Scotland
} 


\subsection{The risk of OSA across the UK}

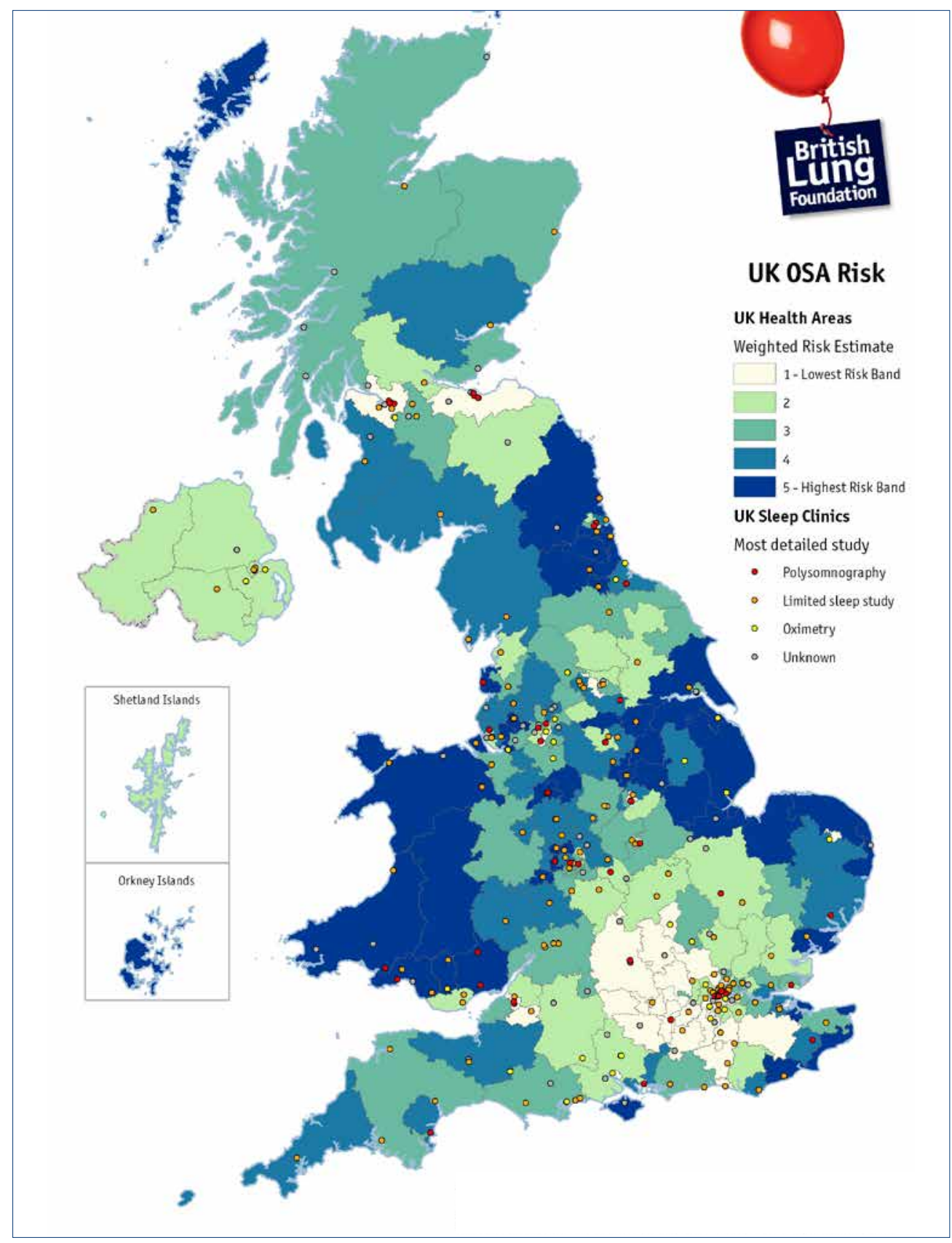

Figure two: UK OSA risk

The relative risk of OSA across the UK has recently been estimated for the first time. ${ }^{19}$ Local population datasets were used to calculate relative risk for each of the health areas across the four nations (Clinical Commissioning Groups (CCGs) in England, Health and Social Care Trusts (HSCTs) in Northern Ireland, NHS Health Boards (HBs) in Scotland and Local Health Boards (LHBs) in Wales).
Estimates of the relative prevalence of OSA in the UK were calculated, based on the geographical distribution of the five risk factors commonly associated with OSA: obesity, gender, age, hypertension and diabetes. The risk factors were weighted according to what the evidence suggests is their relative impact on OSA. 
The map illustrates risk of OSA by quintile, where quintile one is the lowest risk and quintile five is the highest. It shows us that there is significant regional variation in predicted prevalence estimates for OSA. NB it must be taken into account that in built-up areas there will be greater population density; therefore the actual numbers of people with OSA in these areas may be higher than the numbers in more rural areas.

The map shows the locations of known NHS sleep clinics, and colour codes them according to the type of sleep study undertaken there if known. The total number of identified sleep units was 289. There were large differences in the number of available sleep centres per health area, ranging from no sleep centres in 66 health areas to 9 in one large urban area. Some people in areas without sleep centres live close to an urban area and therefore have a short travel distance to access sleep services. Others, specifically in more rural areas or islands, where the population tends to be older and more likely to have OSA, have far greater distances to travel.

\subsection{The cost of OSA - health economics}

There are a number of treatments available for OSA patients at different levels of severity (mild, moderate and severe). The National Institute for Health and Care Excellence (NICE) appraised the use of continuous positive airway pressure (CPAP) compared to lifestyle management and dental devices for the treatment of adults with OSA. ${ }^{20}$ Treating OSA with CPAP was found to be costeffective, as it reduces daytime sleepiness and also offers good value for money. Its cost effectiveness was estimated to be below $£ 5,000$ per qualityadjusted life-year gained (QALY) which is lower than many other interventions recommended by NICE and NICE's incremental cost effectiveness ratio threshold range $(£ 20,000$ to $£ 30,000) .^{21}$

Research published in 2008 estimated that using CPAP over a period of 14 years could result in savings to the NHS close to $£ 1,000$ per patient, and health benefits to patients, including reduction in risks of strokes, cardiovascular events, and road traffic accidents. This led to an increase of the probability of survival after 14 years of treated patients by $25 \%$ as compared to non-treated patients. ${ }^{22}$

The cost savings to the NHS across the UK have been estimated for the first time, using evidence from published research findings. ${ }^{23}$

Annual savings to the NHS in the UK would total $£ 55$ million and 40,000 QALYs, if all people with moderate to severe OSA were to be diagnosed and treated, relative to none being diagnosed and treated.

The estimated annual savings to the NHS in the UK would be $£ 28$ million and 20,000 extra QALYs, if all people with moderate to severe OSA were to be diagnosed and treated, compared with the current estimated level of treatment of 330,000 adults.

\footnotetext{
${ }^{20}$ NICE. 2008a.: Continuous positive airway pressure for the treatment of obstructive sleep apnoea/hypopnoea syndrome. NICE Technology Appraisal guidance 139 ${ }^{21}$ Office of Health Economics 2014: obstructive sleep apnoea health economics report. Consulting report for the BLF

${ }^{22}$ Guest J. F., Helter M. T., Morga A., \& Stradling J. R. 2008: Cost-effectiveness of using continuous positive airway pressure in the treatment of severe obstructive sleep apnoea/hypopnoea syndrome in the UK. Thorax, 63(10), pp.860-865

${ }^{23}$ Office of Health Economics 2014: obstructive sleep apnoea health economics report. Consulting report for the BLF
} 
These estimates of NHS cost savings are due to reductions in consequential acute events (including stroke, cardiovascular events and road accidents) resulting from treatment with CPAP. Treating OSA with CPAP offers greater value for money to the NHS than many other technologies recommended by NICE. According to NICE, the annual number of road traffic accidents could be reduced by one for every 8.5 patients treated with CPAP. ${ }^{24}$ If everyone estimated to have moderate to severe OSA in the UK were treated, this could result in 40,000 fewer road accidents each year relative to the current level of treatment. As some of these accidents result in injury or even fatality, the health gains are considerable.
Treating OSA with CPAP will also lead to benefits from a broader societal perspective, beyond the direct costs to the UK NHS and the health benefits to OSA patients. The evidence suggests that treating OSA with CPAP improves patients' productivity at work and could reduce the cost of work-related injuries to the UK society by $£ 491$ million. ${ }^{25}$ Treating OSA also improves the quality of life of the bed partners of people with OSA.

To summarise, treating everyone with moderate to severe OSA in the UK could double the amount of cost savings to the NHS compared with savings already achieved with current treatment levels, lead to 40,000 fewer road collisions, and achieve 20,000 quality adjusted life years compared with current QALY gains.

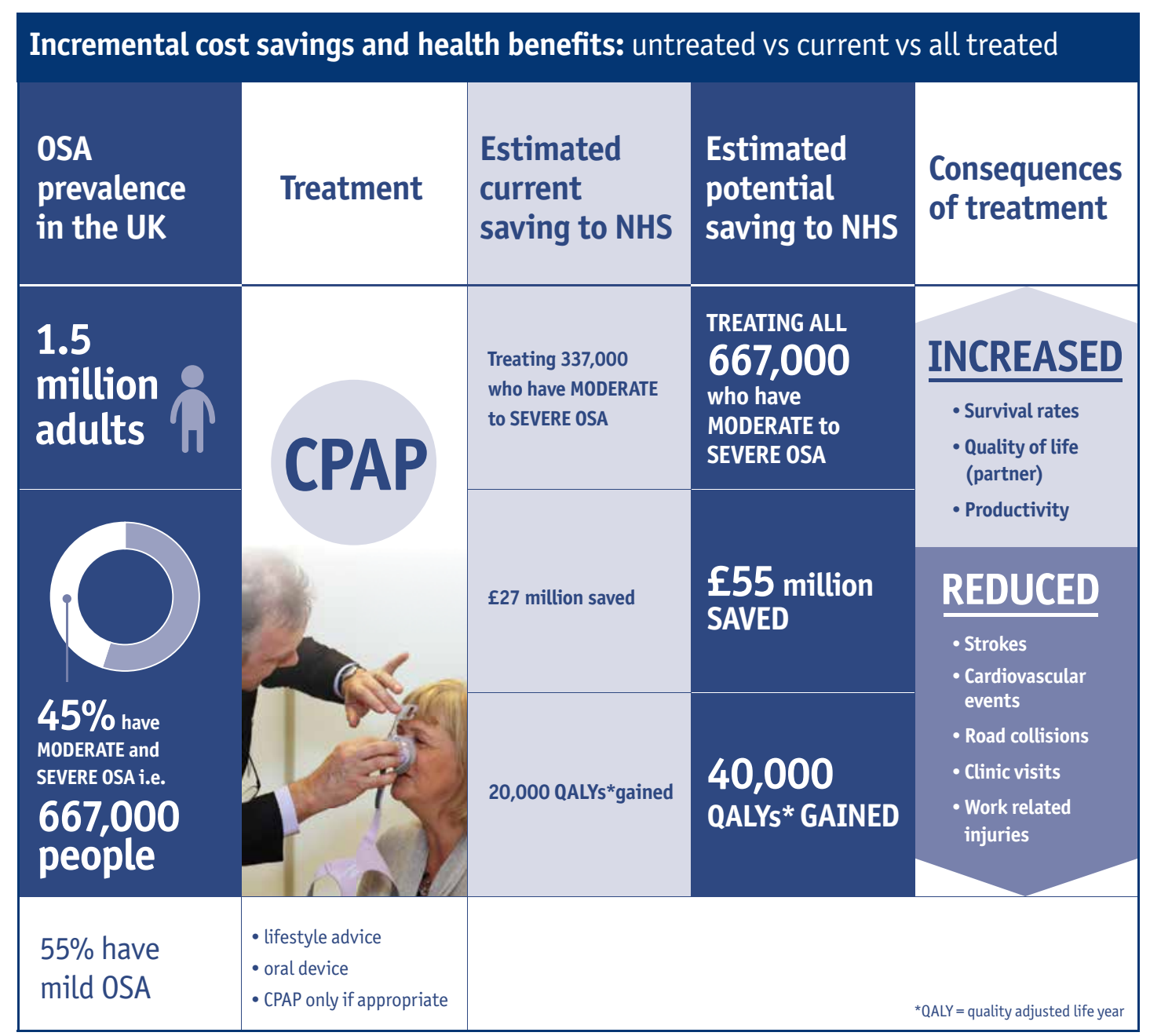

Figure three:

Health

economics and consequences of treating OSA

prevalence

in the UK

1.5 adults

${ }^{24} \mathrm{NICE}, 2008 \mathrm{~b}$. Sleep apnoea - continuous positive airway pressure (CPAP): cost template

${ }^{25}$ Office of health economics 2014: obstructive sleep apnoea health economics report. Consulting report for the BLF 


\section{Service provision and planning}

\subsection{The patient pathway - recommended OSA service provision}

\section{What is in this section?}

This section brings together existing recommendations in guidelines and findings from the BLF's own research since 2011. It follows the patient pathway from assessment and diagnosis through to treatment, management and followup. For each element of the pathway, there is a discussion about service provision, followed by recommendations for the commissioning and service planning process. All the recommendations are collated into the audit tool at the end of this chapter.

\section{Summary of the OSA service pathway}

Figure four provides some of the key elements to be considered in planning services, which are described in detail in this chapter.

Figure four: the OSA service pathway

\begin{tabular}{|c|c|}
\hline Assessment & $\begin{array}{l}\text { - Involvement of GPs } \\
\text { - Referral protocols between primary, secondary and tertiary care } \\
\text { - Components: objective, subjective, physical examination, history, CPAP trial } \\
\text { - Fast track vocational drivers }\end{array}$ \\
\hline Diagnosis & $\begin{array}{l}\text { - Hub and spoke model: roles of tertiary, secondary and primary care } \\
\text { - One stop shop for diagnosis and treatment set-up } \\
\text { - Diagnosis by trained and experienced personnel } \\
\text { - Informing the DVLA } \\
\text { - Patient information } \\
\text { - Data management }\end{array}$ \\
\hline Treatment & $\begin{array}{l}\text { - Patient education about treatment and set-up } \\
\text { - Advice about driving } \\
\text { - Advice about lifestyle } \\
\text { - Data management }\end{array}$ \\
\hline Management & $\begin{array}{l}\text { - Follow-up offered to all patients } \\
\text { - Compliance with therapy } \\
\text { - Replacement of devices and components } \\
\text { - Involvement of other parties } \\
\text { - Data management } \\
\text { - Patient outcomes }\end{array}$ \\
\hline
\end{tabular}




\section{Assessment and diagnosis}

The key components to a good assessment service are a good quality referral, a subjective assessment of sleepiness, a sleep study and a clinical assessment. ${ }^{26}$

In the UK, some clinics also use a trial of CPAP therapy to confirm diagnosis.

Differential diagnosis - Other conditions can cause or contribute to, daytime sleepiness. Misdiagnosis and inappropriate provision of CPAP is wasteful economically and potentially harmful to patients. Differential diagnosis includes the distinction from central sleep apnoea and the recognition of patients with chronic respiratory failure, due, for example, to obesity hypoventilation syndrome or coexistent COPD, as they may require alternative treatment such as non-invasive ventilation. ${ }^{27}$

\section{Components of assessment}

Objective measurement - this is in the form of an overnight sleep study. The most simple and cheapest study uses pulse oximetry. Oximetry alone can confirm OSA but has significant rates of both false negatives and false positives. ${ }^{28}$ Some sleep clinics use a multi-channel portable system which gives more information, such as chest movement and nasal airflow. Both pulse oximetry and multichannel devices can be used at home. Historically, polysomnography (PSG) was considered the gold standard for the confirmation of an OSA diagnosis. However increasingly in the UK and elsewhere, PSG, which has to be undertaken in a hospital setting, is used only for more complex cases or where simpler tests have proved inconclusive. This has been confirmed by research findings. ${ }^{29} 30$
Subjective assessment - this is used to assess the patient's levels of sleepiness and other symptoms which affect their daily living. The most widely used subjective tool is probably the Epworth Sleepiness Scale, although other tools are also used, such as the OSA 50 and the STOP Bang questionnaire. The Epworth Sleepiness Scale is available from the BLF website: www.blf.org.uk/Page/

\section{Epworth-Sleepiness-Scale}

\section{Physical examination and clinical history - this includes medical history, weight and height measurements, and blood pressure. Patients may also be asked to perform some breathing tests and there may be an examination of the upper airway.}

CPAP trial - Continuous positive airway pressure is sometimes offered on a trial basis to confirm the diagnosis.

\section{Referral pathways}

Referral may come from a GP, from a hospital colleague, or may be included in a pre-assessment clinic, e.g. for bariatric surgery. This will vary across the UK, depending on what services are available where.

\section{The role of GPs in assessment of OSA}

It has long been reported that the recognition and diagnosis of OSA would be improved if GPs were trained or reminded to consider the possibility of OSA ${ }^{31}$ and various studies show that there is a clear failure to suspect the incidence of OSA in primary care. ${ }^{32}$

\footnotetext{
${ }^{26}$ BLF 2014: OSA conference report

${ }^{27}$ IMPRESS 2009: Service specification for investigation and treatment of obstructive sleep apnoea syndrome

${ }^{28}$ IMPRESS 2009: Service specification for investigation and treatment of obstructive sleep apnoea syndrome

${ }^{29}$ Whittle A.T., Finch S.P., Mortimore I.L., MacKay T.W., Douglas N.J. 1997: Use of home sleep studies for diagnosis of the sleep apnoea/hypopnoea syndrome.

Thorax 1997;52:1068-1073

${ }^{30}$ William A. Whitelaw, Rollin F. Brant, and W. Ward Flemons 2005: Clinical usefulness of home oximetry compared with polysomnography for assessment of sleep apnea Am J Respir Crit Care Med Vol 171. pp 188-193, 2005

${ }^{31}$ IMPRESS 2009: Service specification for investigation and treatment of obstructive sleep apnoea syndrome

${ }^{32}$ M.A. Martínez-García P. Catala'́n Serra 2010: Breathe December Volume 7 No 2
} 
Models are developing in some parts of the UK where GPs and their colleagues in secondary care develop a local service specification for assessment and diagnosis, with the respective roles agreed and implemented across the local area. In some areas, GPs have gone a step further and now offer overnight sleep studies to patients. There are a few models for this already in place, depending on local expertise and interest.

\section{See appendix 5.1 for a referral protocol template based on an existing model}

A recent study showed that a simple low cost case finding and management program in primary care, which is focused on patients with obesity, hypertension and diabetes, can work well in finding undiagnosed OSA. ${ }^{33}$

Over time, it is envisaged that the role of GPs in the assessment of OSA will continue to develop as it has in other conditions (e.g. asthma and hypertension).

\section{Driving and OSA}

We have already highlighted that people whose work involves driving are particularly at risk if they have undiagnosed OSA. The BLF has been advocating the fast-tracking of vocational drivers since 2011, and supports the OSA partnership's four week wait campaign. ${ }^{34}$

\section{Assessment recommendations}

- The local referral pathway should include all referral routes between primary, secondary and tertiary services (GPs, hospital colleagues, other clinics)

- GPs should be adequately supported and trained to recognise the symptoms of OSA

- GP practices should be encouraged to take part in case finding programmes for undiagnosed OSA in high risk patients
- GP practices should carry out screening for OSA in symptomatic patients, including using a subjective assessment of sleepiness and clinical history

Sleep clinics and health areas* should explore setting up local shared models (primary and secondary care) for the assessment of OSA in the local population, including a referral protocol with a referral letter or online template

Assessment should include an overnight sleep study, a subjective measure of sleepiness, a clinical history and a physical examination

- The assessment of vocational drivers with obstructive sleep apnoea (OSA) who are sleepy should be expedited to allow driving again within a maximum of four weeks following first referral

- Fast tracking should also be considered for people employed in other vigilance critical roles

A regional service specification should be developed which incorporates responses to all of the above assessment recommendations

*(Clinical Commissioning Groups (CCGs) in England, Health and Social Care Trusts (HSCTs) in Northern Ireland, NHS Health Boards (HBs) in Scotland and Local Health Boards (LHBs) in Wales)

\section{Diagnosis}

The most common feeling experienced by patients at diagnosis is relief. ${ }^{35}$

Traditionally, the role of assessment and diagnosis lay firmly in secondary care. As we have discussed, the role of primary care in the assessment of OSA could be developed. However, OSA specialists continue to advise that the diagnosis of OSA should be made by an experienced health care professional in secondary care.

\footnotetext{
${ }^{33}$ Burgess K.R., Havryk A., Newton S. et al 2013: Targeted case finding for OSA within the primary care setting J Clin Sleep Med 2013;9(7):681-686

${ }^{34}$ OSA partnership 2014: Four week wait campaign for the treatment of obstructive sleep apnoea syndrome (OSAS) for vocational drivers

${ }^{35}$ BLF 2013: OSA patient experience survey
} 
The Association of Respiratory Technology and Physiology (ARTP) have produced Standards of care for OSA services (diagnostics). This document aims to outline minimum standards together with a code of conduct that will protect patients and maintain high standards of sleep diagnostic services, whoever delivers them. ${ }^{36}$ There are eleven standards, which are explained in detail in the document, but which are summarised here:

All patients who have the symptoms of obstructive sleep apnoea can expect the following level of care within the United Kingdom:

1. They will be referred to a specialist service which will undertake a sleep study which as a minimum will measure respiratory signal and arousal signal from sleep signals (e.g. overnight oximetry and upwards). Negative studies should either be repeated or escalated up to more advanced diagnostics (e.g. multi-channel studies) in patients with significant symptoms.

2. They will have their signs, symptoms and screening sleep study reviewed by an OSA expert who will have sufficient experience, training and qualifications to provide such an independent professional opinion or a differential diagnosis.

3. They should expect to receive a treatment pathway which will give them appropriate treatment for the severity of their OSA and in accordance with any co- morbidities (cardio-vascular disease, heart failure, stroke, obesity, diabetes or other respiratory disease such as asthma, or COPD).

4. They should have a titration study to determine whether (a) CPAP is beneficial and (b) at what level it is optimally beneficial as well as to (c) determine whether the therapy is tolerated. Single night or split night titration studies are not recommended.

5. They should be issued or trialled with CPAP with a review of the intervention within 4 weeks of continual use of the therapy either by remote monitoring or a sleep study seen by an independent expert in sleep medicine who will have sufficient experience, training and qualifications to provide such an opinion.
6. They should have continuous access to clinical support and further diagnostic tests throughout their period of treatment.

7. Patients should have rapid access to an expert in OSA and sleep medicine who can review their clinical situation and provide advice or improved treatment for their OSA or referral to an appropriate specialist.

8. They should be seen by a healthcare expert in sleep who can advise on all aspects of OSA interventions including lifestyle changes, dieting and weight loss, exercise programmes, ENT surgery or assessments, differential diagnosis, justification for treatment and alternative treatments (nasal prongs, mandibular advancement devices, other care).

9. All diagnostic services should be provided on "value added" basis which is best for the patient - and should not be driven by cost/price.

10. All services should adhere to the ARTP Standards of Care for OSA Services.

11. OSA services should be delivered from centres which fulfil criteria which include:

a. Safe, clean, environment with patient privacy and access to high quality services b. Taking due regard of every patient's right to privacy, confidentiality and dignity

c. Appropriate protection of data of all patient information and files d. Offering an appropriate selection of CPAP devices and patient interfaces as well as an array of other therapeutic interventions

e. Centres staffed with trained and experienced personnel in diagnostics and therapeutics for OSA $f$. Centres offering a variety of diagnostics for OSA from simple screening to complex multi-channel devices

g. Provision of a summary report of sleep studies in an easily understandable and readable format written in plain English and explaining technical/ medical terms where required h. Provision of reports to general practitioners and relevant specialists in a timely manner. 
The IMPRESS service specification also recommends that diagnosis requires specialist clinical experience and depends on the combination of the clinical history and the result of a sleep study. ${ }^{37}$ The specification also recommends the availability of an experienced specialist multidisciplinary team, comprising medical, nursing and scientific or technical specialists. There are various models available for the diagnosis of OSA, but the most important factor is the experience of the personnel. The role of a clinical lead is seen as particularly important.

The role of the lead for the team includes:

- clinical leadership, service development and innovation

- clinical assessment

- recognition of relevant co-morbidities

- interpretation of the sleep study in the context of the overall clinical picture

- diagnosis (and differential diagnosis)

- decisions on treatment

- advice to other members of the team and provision of reports to relevant external agencies (e.g. occupational health, DVLA) ${ }^{38}$

Some clinics offer a one stop shop approach to diagnosis which saves time for the clinic and the patients, and which, if managed efficiently, cuts down on waiting times. One such model uses a referral and sleep study pathway in partnership with the local GP surgery. The results are reported and then the patient is seen in clinic for their results, and to be set up on a trial of CPAP treatment the same day. They return three weeks later for a review. ${ }^{39}$
It is recommended that regional service specifications are developed in order to confirm in writing the agreed model and to details all aspects of the service. ${ }^{40}$

Hub and spoke models are in place in some areas where there is local agreement between the primary, secondary and tertiary service about the roles and responsibilities of each. One example is the regional service that has been developed in the North West of England. ${ }^{41}$

It is recommended that those involved in diagnosis should take part in programmes to develop and improve expertise in diagnostic test analysis. ${ }^{42}$

There should be protocols in place to ensure consistent messaging around advising and supporting patients about informing the DVLA. Some existing guidelines include template letters ${ }^{43}$ and the British Thoracic Society have written a position statement on this. ${ }^{44}$

\section{Diagnosis recommendations}

A local hub and spoke model for diagnosis of OSA should be agreed between tertiary, secondary and primary care to ensure that all people living in the local community have equal access to a diagnostic service

- There should be mechanisms in place to minimize waiting times for patients at the diagnostic stage in secondary care, such as a one stop shop for diagnosis and treatment set-up

- The local model should specify the agreed objective and subjective assessment and diagnostic interventions that will be used

- The local model should be shared amongst all relevant stakeholders

\footnotetext{
${ }^{37}$ IMPRESS 2009: Service specification for investigation and treatment of obstructive sleep apnoea syndrome

${ }^{38}$ Mackay T. 2010: Working towards the development of minimal standards for referral, investigation and treatment in Scotland

${ }^{39}$ BLF 2014: OSA conference report

${ }^{40}$ BLF 2014: OSA conference report

${ }^{41}$ North of England Specialist Commissioning Group (NW office) 2012: Sleep-related breathing disorders

${ }^{42}$ Mackay T. 2010: OSA working towards the development of minimal standards for referral, investigation and treatment in Scotland

${ }^{43}$ Mackay T. 2010: OSA working towards the development of minimal standards for referral, investigations and treatment in Scotland

${ }^{44}$ BTS 2014: Position statement on driving and OSA
} 
- Diagnosis of OSA should be confirmed by a trained and experienced sleep specialist

- At diagnosis, patients should be given high quality information about OSA, such as the BLF OSA leaflet

- Drivers should be told about informing the DVLA and there should be procedures in place to ensure consistency of approach

- Vocational drivers diagnosed with OSA should have access to treatment so that they can return to driving within four weeks of first referral

- All regions should provide a hospital-based tertiary specialist sleep service, including facilities for full polysomnography, for the assessment of more complex cases, or where a simple sleep study is inconclusive ${ }^{47}$

- Staff involved in diagnosing OSA should take part in regular training and education programmes to develop and improve expertise

- A regional service specification should be developed which incorporates responses to all of the above diagnosis recommendations

- The Association of Respiratory Technology and Physiology (ARTP) Standards of care for OSA Services (Diagnostics) should be followed

\section{Treatment}

"I woke up feeling like a million dollars."

"CPAP is my best friend."

"Getting diagnosed and treated changed my life, and more importantly my wife's."

Treatments for OSA aim to reduce daytime sleepiness, improve quality of life, and reduce the risk of health complications and accidents. Treatment consists of ways to reduce the number of apnoeas and hypopnoeas during sleep (caused by blockages and part-blockages of the airway).

Continuous Positive Airway Pressure (CPAP) is recommended for adults with moderate or severe symptomatic OSA. It is only recommended for patients with mild OSA if they have symptoms affecting their quality of life, and where lifestyle advice and other treatment options have been unsuccessful or are considered inappropriate. ${ }^{48}$

The IMPRESS service specification recommends the following:

- Facilities, equipment and experienced personnel necessary to initiate CPAP treatment and train patients

- Availability of a wide range of interfaces to allow provision of the most appropriate CPAP machine and interface, plus humidifier if required

- A database of patients receiving CPAP, to include equipment type, interface, settings, service history, symptom control and compliance ${ }^{49}$

In addition, the ARTP standards of care for OSA diagnostics, as already described in the diagnosis section of this booklet, specify recommend skill levels and protocols to ensure quality and safety of provision. ${ }^{50}$

\footnotetext{
${ }^{45}$ BLF 2012: OSA patient charter

${ }^{46}$ National Institute for Health and Clinical Excellence (NICE) technology appraisal guidance 139 2008: Continuous positive airway pressure for the treatment of obstructive sleep apnoea/hypopnoea syndrome

${ }^{47}$ IMPRESS 2009: Service specification for investigation and treatment of obstructive sleep apnoea syndrome

${ }^{48}$ ARTP 2010: Standards of care for sleep apnoea services (diagnostics)
} 
In the BLF OSA patient survey of nearly 3,000 people with OSA in the UK, $75 \%$ of people were positive about their treatment. Continuous positive airway pressure (CPAP) was given to $92 \%$ of patients. Initial treatment had a positive effect for $77 \%$ of those treated with CPAP. Being diagnosed and treated for OSA had a highly significant positive effect on all seven aspects of wellbeing that were assessed. Improvements in wellbeing following treatment were greater for: those whose OSA was more severe at the time of diagnosis; those who reported positive emotions at the time of diagnosis (compared with those who reported negative emotions); for younger people; for those with a weight problem; and for those who used CPAP for more hours per night. ${ }^{49}$

CPAP is a lifetime commitment for most patients. It can be hard to get used to, and several factors can affect whether people persevere with treatment. These include having the correct settings on the machine, having the correct components such as the mask and tubing, and receiving the right support and education. Long term patterns of CPAP use are established in the first week of treatment. ${ }^{50}$ It has been shown that CPAP use and outcomes of therapy can be improved by the provision of a nurse-led intensive CPAP education and support programme, ${ }^{51}$ and although this level of service may not be feasible in every setting, it highlights the importance of CPAP education and support. Also, this service can be a physiologist led service.

The value of Mandibular Repositioning Device (MRD) therapy in the treatment of mild/moderate OSA has been recognised. There are a variety of custom manufactured MRD designs available. Some designs may be deemed more appropriate for the individual patient after assessment. It is recommended that these devices are provided as part of a service which is delivered by experienced dental practitioners in the field of dental sleep medicine who have undergone documented training. ${ }^{52}$
Lifestyle changes, such as weight loss, sleep routines and avoiding certain substances (alcohol, caffeine, nicotine and sedatives) are also recommended as an important addition to treatment with medical devices. ${ }^{53}$

\section{Treatment recommendations}

CPAP treatment should be available to all local people with moderate to severe OSA, as recommended by NICE

- At treatment set-up, all patients should take part in training and education to ensure they understand how to use their equipment and what it is for

- A range of CPAP interfaces should be available, including access to humidification

Clear advice should be offered to patients who drive about informing the DVLA

- Mandibular repositioning devices should be available on the NHS to people with mild to moderate OSA, with access to appropriately trained personnel for fitting these devices

- Lifestyle advice should be offered routinely as part of treatment for OSA

- A local database should provide up to date and accurate information about patients referred, patients diagnosed with OSA, and which treatment these patients are using

- A regional service specification should be developed which incorporates responses to all of the above treatment recommendations
${ }^{49}$ BLF 2013: OSA patient experience survey
${ }^{50}$ BLF 2014: OSA conference report
${ }^{51}$ Hoy C.J., Vennelle M., Kingshott R.N., Engleman H.M., Douglas N.J. 1999:
Can intensive support improve CPAP use in patients with the SAHS?
Am J Respir Crit Care Med
${ }^{52}$ ARTP 2011: Standards of care for mandibular repositioning devices
${ }^{53}$ BLF website 2014 


\section{Management}

\section{Monitoring and follow-up}

Regular monitoring and follow-up of patients is recommended. This includes:

- Open clinic access for CPAP-related problems or telephone support line

- Provision of replacement machines and parts as required

- Monitoring of patient compliance, symptoms and side-effects of treatment

- Providing advice and recommendations on alternative or adjunctive treatment ${ }^{54}$

Many clinics offer regular face to face followup appointments. However, with the growing numbers of patients on CPAP, clinics are now facing "coping with the CPAP mountain". ${ }^{55}$ Some clinics are resorting to discharging their patients due to inadequate resources, especially patients who are assessed as "low risk" and stable on CPAP, who are offered open access to an advice helpline and to a service providing replacement masks and parts as necessary. ${ }^{56}$

The Primary Care Respiratory Society (PCRS-UK) opinion sheet on OSA recommends that shared care of patient follow-up, advice on lifestyle management and management of cardio-metabolic and other co-morbidity is appropriate. ${ }^{57}$

A management model that is being used in some areas involves working in partnership with medical device companies. This can be a pragmatic solution if there are robust systems and protocols in place to ensure quality and safety.
This may be implemented

- in the initial weeks following CPAP set-up, as the long term pattern of use is established in the first week, or

- for the ongoing monitoring of CPAP compliance.

Some clinicians feel that this is a positive way forwards as it reduces the burden on the sleep clinic to provide all aspects on ongoing management directly. Patients are still referred back to the sleep clinics if there are any issues. This approach may be particularly useful in rural areas, where patients may have to travel many miles to their nearest sleep clinic. $^{58}$

Results of the BLF's survey of people with OSA indicated that most people wanted future services for assessment, diagnosis, treatment and ongoing support to be located either in a local specialist sleep centre or in their local hospital..$^{59}$ However, these responses may have been affected by the fact that most of the participants were already receiving the service in this way and would like it to remain the same. Less than $1 \%$ said that they wanted telehealth services for all aspects of care, except for ongoing support, which just over $1 \%$ indicated as a preference. Therefore any change in existing service would need to be planned and delivered carefully.

The ARTP 2010: Standards of Care for Sleep Apnoea Services (CPAP) set the standards for a detailed CPAP service. The standards apply not only to the equipment (CPAP devices and interfaces) but also to the general services, including diagnostic assessments, workforce, training and related issues. ${ }^{60}$

\footnotetext{
${ }^{54}$ IMPRESS 2009: Service specification for investigation and treatment of obstructive sleep apnoea syndrome

${ }^{55}$ BLF 2014: OSA conference report

${ }^{56}$ Mackay T 2010: Working towards the development of minimal standards for referral, investigation and treatment in Scotland

${ }^{57}$ PCRS-UK 2013: Opinion sheet No. 53 - Sleep Apnoea

${ }^{58}$ BLF 2014: OSA conference report

${ }^{59}$ BLF 2013: OSA patient survey

${ }^{60}$ ARTP 2010: Standards of care for sleep apnoea services (CPAP)
} 


\section{Partnerships}

Management of OSA by a sleep service should also include pathways with / to other services:

- Onward referral for more specialised investigation where clinically appropriate

- Reporting to the patient's registered GP and secondary care specialist(s) following each patient contact (other than parts provision)

- Providing advice and reports for other agencies e.g. occupational health services and DVLA regarding medical fitness for work and driving

- Close liaison or integration with services which share similar expertise and technology (e.g. long term domiciliary, NIV)

- Ready access to, and cooperation with, clinical services dealing with the common co-morbidities of OSA (obesity, diabetes, cardiovascular disease, ENT conditions) ${ }^{61}$

\section{Data management}

Collating, updating and reviewing data is an important element of the service, including:

- A database of patients receiving CPAP, to include equipment type, interface, settings, service history and compliance

- Documenting pathways of care for patients

- Data on patient related outcome measures in accordance with agreed audit criteria

- Accurate identification of those patients who are not using their CPAP machines so that these machines can then be reclaimed and recycled

- Reports for monitoring and informing commissioning / service planning decisions

- Allow comparisons of subgroups e.g. age, sex, BMl, severity

- Facilitate service improvements locally and nationally, by providing robust comparable data
- Monitor the effects of changes in practice

- Facilitate clinical research ${ }^{6263}$

See appendix 5.2 for suggested list of key performance indicators - outputs and outcomes to measure

\section{Patient experience and outcomes}

It is recommended that clinics in the local health area carry out patient experience surveys annually to help inform their service and that the results of these are collated at a health area level. The BLF carried out a patient experience survey in 2013 and collated the views of nearly 3,000 patients across the UK.

See appendix 5.3 for the BLF patient survey questionnaire template

\section{Management recommendations}

- Follow-up after diagnosis and treatment should be offered to all patients

- Face to face follow up should be available to those who need it, for example vocational drivers, complex cases

- All patients should have access to help and support by telephone

- Medical devices and components should be replaced as required

- The local model should include onward referral and partnership working arrangements with relevant clinical services

- The local database should provide up to date and accurate information about treatment compliance and follow up

A regional service specification should be developed which incorporates responses to all of the above management recommendations

Patient experience / satisfaction surveys should be performed annually to inform service planning 


\subsection{Planning / commissioning - recommended process}

\section{Regional service specification}

A successful model for developing sleep services is one which demonstrates the need, looks at current service provision, identifies what services should be in place, and offers a proposed structure. A regional specification has been developed in the north west of England, which is available on request. ${ }^{64}$

\section{Demonstrate the need}

This involves highlighting the demand for the service, explaining the condition, its prevalence and impact, quoting relevant guidelines and recommended treatments, identifying barriers and challenges, stating the case for local pathways, and explaining the health economics.

\section{Look at current services}

This needs to include identifying the current roles of different health care professionals and partner organisations / stakeholders.

\section{Examine what services should be available}

This involves identifying what additional capacity and capability would include, such as infrastructure, staffing, training, treatment, access to comprehensive and specialist services. It also involves using a network approach involving primary, secondary and tertiary care.

\section{Confirm the proposed solution}

This should be based on current guidelines and local need. It should include the roles of all those in the local network, including primary care, secondary care and tertiary care, and should give detail about the resource requirements, training requirements, key performance indicators, potential funding streams, and locally agreed fees. ${ }^{65}$

\section{Recommended staffing - experience and expertise}

ARTP recommend the service is provided by experienced trained personnel with qualifications or a suitable period of appropriate supervised practice with a nationally registered healthcare professional and with adequate and up to date experience in sleep. These would include:

- Consultant Specialist Physician/ENT Surgeon (Registered with GMC and member of a Royal College)

- Registered Respiratory Nurse Specialist (Registered with NMC)

- Registered Physiotherapist (Registered with HPC)

- Registered Healthcare Scientist (Registered with HPC or RCCP)

- GP with training and competence who has a special interest (GPWSI) in OSA

Healthcare professionals in Band 7 or above with over 24 months appropriate experience in OSA services should be able to assess the patient's clinical picture and recommend CPAP trials (and possibly issue CPAP). Usually such staff will be working to an agreed protocol designed in conjunction with the medical team. ${ }^{66}$ The IMPRESS service specification staffing recommendations of 2009 were reviewed by a panel of OSA clinicians in 2014, for this toolkit. They concluded that the staffing levels should be amended.

\section{Updated recommendations for staffing an established OSA diagnostic, treatment and management service \\ Points to bear in mind:}

- The amount of medical time required may be less than recommended here if there are trained and skilled technical/nursing staff available

- A new service could start with lower staffing levels than those recommended here, and build up if demand outweighed capacity over time

- As the service grows, there may be a need for a change in staffing levels, or the need to look at provision of some elements of the work with partner organisations

\footnotetext{
${ }^{64}$ North of England Specialist Commissioning Group (NW office) 2012: sleep-related breathing disorders 
- In some areas, the number of referrals may be higher due to local services e.g. bariatric services, and local prevalence of associated risk factors such as obesity, hypertension, and diabetes

- It is up to local areas to identify the exact make-up of the team depending on the local population, and to decide which service the sleep service sits within, or if it is set up as a separate service

- Some clinics offering a "full" sleep service will have a multi-disciplinary team or network to deliver services, which may include staff from a respiratory, neurology, dentistry, dietitian, or ENT background. This tool does not specify which discipline staff should be from

- These recommendations are for a service that is already up and running, has been established for four years, and which serves a population of 500,000

- For a population of 500,000, at current levels of referral, it is estimated that there will be approximately 1,000 referrals with the introduction of around 500 CPAP's. Over four years this would accrue to around 2,000 patients requiring on-going management

\section{Consultant/medical time}

Assuming a consultant works for 42 weeks and will see six new patients in a clinic there is a requirement for 166 clinics, i.e. four new patient clinics a week.

There will be a need for some consultant activity from the follow ups and the reports, and these would require two additional clinics a week i.e. a total of six programmed activities (PAs).

The amount of medical time required may be less if there are trained and skilled technical/ nursing staff available.

\section{Nursing / technical time}

There is a need to provide governance for patients already established on CPAP who are reviewed by nursing / technical staff. The Royal College of Physicians state that one direct clinical care (DCC) is needed per 50 patients on ventilators.
While no such document exists for CPAP it would seem appropriate to say, as a minimum, that one DCC is needed per 500 CPAP patients. Assuming that a clinic will have 2,000 patients on CPAP this equates to four DCC of activity to support the technical / nursing staff.

\section{Recommended staffing levels for an established service (four years)}

\section{Medical infrastructure (consultant)}

\begin{tabular}{|l|l|}
\hline Activity & $\begin{array}{l}\text { Number of programmed } \\
\text { activities }\end{array}$ \\
\hline $\begin{array}{l}\text { New / follow-up } \\
\text { patient clinics }\end{array}$ & $6^{*}$ \\
\hline $\begin{array}{l}\text { Administration time } \\
\text { for clinics (DCC) }\end{array}$ & 1 \\
\hline $\begin{array}{l}\text { Reporting time for } \\
\text { sleep studies }\end{array}$ & 2 \\
\hline Service development & 1 SPA* $^{* *}$ \\
\hline $\begin{array}{l}\text { Governance for } \\
\text { patients on CPAP }\end{array}$ & 4 \\
\hline Total & 14 \\
\hline
\end{tabular}

*The amount of medical time required may be less if there are trained and skilled technical/nursing staff available

** SPA = supporting professional activities

\begin{tabular}{|l|l|l|}
\hline \multicolumn{2}{|l|}{ Administration } & \multicolumn{1}{l|}{} \\
\hline 1.4 WTE & Band 4 & Co-ordinator \\
\hline 1 WTE & Band 3 & Typist \\
\hline 1 WTE & Band 3 & $\begin{array}{l}\text { Typist / general } \\
\text { administrator / } \\
\text { maintain database. }\end{array}$ \\
\hline
\end{tabular}

This level of support would exclude the general clinic office administration of booking appointments and undertaking routine activities that would take place normally in an outpatient setting. 


\begin{tabular}{|l|l|l|}
\hline \multicolumn{2}{|l|}{ Technical / nursing infrastructure } \\
\hline 1 WTE & Band 8a / 7 & $\begin{array}{l}\text { Manager and senior } \\
\text { reporter undertaking } \\
\text { clinics / new CPAP /returns }\end{array}$ \\
\hline 3 WTE & Band 6 & $\begin{array}{l}\text { Reporting / undertaking } \\
\text { clinics / CPAP initiations } \\
\text { (training) }\end{array}$ \\
\hline 2 WTE & Band 2 / 3 & $\begin{array}{l}\text { Support clinics / issue } \\
\text { equipment / support } \\
\text { helpline / stock control }\end{array}$ \\
\hline
\end{tabular}

The OSA calculator (tool B of this toolkit), calculates the staffing recommended for a health area using these recommendations, taking into account the actual population figures of that area and the estimated OSA prevalence for an area of this size.

\section{Regional service specification recommendations}

- Services are available or accessible for the assessment, diagnosis, treatment and management of OSA

OSA should be included in relevant strategic plans
- A regional service specification should be in developed and in place which includes details about the roles of primary, secondary and tertiary care, and which covers all aspects of assessment, diagnosis, treatment and management of OSA in the local population

- The service specification should give details of resource requirements, training requirements, key performance indicators, funding and fees (if appropriate)

- The local OSA service in each hospital should have a named clinical lead (often medical)

- Staffing levels, expertise and experience should be in line with those recommended in the BLF OSA toolkit and by ARTP

- A multi-disciplinary team should be involved, consisting of medical, nursing, scientific or technical experts, plus access to other health care professionals e.g. bariatric surgery, ENT, dental, psychology, dietician

A list of recommendations is drawn up following a service audit and shared as appropriate

\subsection{Audit and calculator tools}

The two tools that have been developed should be used in conjunction with the information contained in this booklet.

\section{Tool A: Reviewing local services for OSA:}

\section{Audit checklist}

Looking at current services - The audit tool can be used to carry out a review of current provision, repeated at regular intervals. It lists the recommendations in this booklet, so that service planners and commissioners can ascertain current levels of service and identify actions. Links to relevant supporting documentation are provided.
Tool B: Planning local OSA services:

OSA service calculator

Demonstrating the need, examining what services should be available, the proposed

solution - The calculator tool can be used to help with these decisions. The tool generates a report for a specific health area (CCGs in England, HSCTs in Northern Ireland, NHS HBs in Scotland and LHBs in Wales). The report provides information on the adult local population, the estimated OSA prevalence, recommended service provision for a population of this size, cost avoidance for treating OSA, and information about the local prevalence of OSA risk factors to consider when making decisions about service provision. 


\section{Tool A: Reviewing local services - OSA audit checklist}

To be completed on a regular (e.g. annually) basis at a regional level.

\section{Date of audit:}

\section{Region:}

\section{Audit conducted by:}

$\mathrm{Y}=$ yes $\quad \mathrm{N}=$ no $\quad ?=$ don't know

\begin{tabular}{|c|c|c|}
\hline Y/N/? & Recommendation & Action needed \\
\hline & PART ONE: REGIONAL SERVICE SPECIFICATIONS & \\
\hline & $\begin{array}{l}\text { Services are available or accessible for the assessment, diagnosis, } \\
\text { treatment and management of OSA }\end{array}$ & \\
\hline & OSA is included in relevant strategic plans & \\
\hline & $\begin{array}{l}\text { A regional service specification includes details about roles of primary, } \\
\text { secondary and tertiary care, and covers assessment, diagnosis, } \\
\text { treatment and management of OSA in the local population }\end{array}$ & \\
\hline & $\begin{array}{l}\text { The service specification gives details of resource requirements, } \\
\text { training requirements, key performance indicators, funding and fees } \\
\text { (if appropriate) }\end{array}$ & \\
\hline & The local OSA service in each hospital has a named clinical lead & \\
\hline & $\begin{array}{l}\text { Staffing levels and experience are in line with those recommended } \\
\text { in the BLF OSA toolkit and by ARTP }\end{array}$ & \\
\hline & $\begin{array}{l}\text { A multi-disciplinary team is involved, including medical, nursing, } \\
\text { scientific or technical experts, plus access to other health care } \\
\text { professionals }\end{array}$ & \\
\hline & $\begin{array}{l}\text { A list of recommendations is drawn up following this audit } \\
\text { and shared as appropriate }\end{array}$ & \\
\hline & PART TWO: ASSESSMENT AND DIAGNOSIS & \\
\hline & $\begin{array}{l}\text { GPs are adequately supported and trained to recognise } \\
\text { the symptoms of OSA }\end{array}$ & \\
\hline & $\begin{array}{l}\text { GP practices are encouraged to take part in case finding programmes } \\
\text { for undiagnosed OSA in high risk patients }\end{array}$ & \\
\hline & $\begin{array}{l}\text { GP practices carry out screening for OSA in symptomatic patients, } \\
\text { including using a subjective assessment of sleepiness and } \\
\text { clinical history }\end{array}$ & \\
\hline & $\begin{array}{l}\text { Local referral pathways include all referral routes - from primary care, } \\
\text { from hospital colleagues and from other clinics }\end{array}$ & \\
\hline & $\begin{array}{l}\text { Local shared models (primary and secondary care) are in place for the } \\
\text { assessment of OSA, including a referral protocol with a referral letter/ } \\
\text { online template }\end{array}$ & \\
\hline & $\begin{array}{l}\text { Assessment includes an overnight sleep study, a subjective measure } \\
\text { of sleepiness, a clinical history and a physical examination }\end{array}$ & \\
\hline & $\begin{array}{l}\text { The assessment of vocational drivers with OSA who are sleepy allows } \\
\text { driving again within a maximum of four weeks following first referral }\end{array}$ & \\
\hline & $\begin{array}{l}\text { Fast tracking is in place for people employed in other } \\
\text { vigilance critical roles }\end{array}$ & \\
\hline
\end{tabular}




\begin{tabular}{|c|c|}
\hline $\begin{array}{l}\text { A local hub and spoke model for diagnosis of OSA is agreed between } \\
\text { tertiary, secondary and primary care to ensure equal access to a } \\
\text { diagnostic service }\end{array}$ & \\
\hline $\begin{array}{l}\text { There are mechanisms in place to minimize waiting times for patients } \\
\text { at the diagnostic stage in secondary care, such as a one stop shop }\end{array}$ & \\
\hline $\begin{array}{l}\text { The local model specifies the objective and subjective assessment and } \\
\text { diagnostic interventions that will be used }\end{array}$ & \\
\hline The local model is shared amongst all relevant stakeholders & \\
\hline $\begin{array}{l}\text { Diagnosis of OSA is confirmed by a trained and experienced } \\
\text { sleep specialist }\end{array}$ & \\
\hline $\begin{array}{l}\text { At diagnosis, patients are given high quality information about OSA, } \\
\text { such as the BLF OSA leaflet }\end{array}$ & \\
\hline $\begin{array}{l}\text { Drivers are told about informing the DVLA and there are } \\
\text { procedures in place to ensure consistency of approach }\end{array}$ & \\
\hline $\begin{array}{l}\text { Vocational drivers diagnosed with OSA have access to treatment } \\
\text { so that they can return to driving within four weeks of first referral }\end{array}$ & \\
\hline $\begin{array}{l}\text { Access to a hospital-based tertiary specialist sleep service, including } \\
\text { facilities for full polysomnography, for the assessment of more } \\
\text { complex cases, or where a simple sleep study is inconclusive }\end{array}$ & \\
\hline $\begin{array}{l}\text { Staff involved in diagnosing OSA take part in regular training and } \\
\text { education programmes to develop and improve expertise }\end{array}$ & \\
\hline $\begin{array}{l}\text { The Association of Respiratory Technology and Physiology (ARTP) } \\
\text { Standards of care for OSA Services (Diagnostics) are followed }\end{array}$ & \\
\hline PART THREE: TREATMENT AND MANAGEMENT & \\
\hline $\begin{array}{l}\text { CPAP treatment is available to all local people with moderate to severe } \\
\text { OSA, as recommended by NICE }\end{array}$ & \\
\hline $\begin{array}{l}\text { At treatment set-up, all patients take part in training and education to } \\
\text { ensure they understand how to use their equipment and what it is for }\end{array}$ & \\
\hline $\begin{array}{l}\text { A range of CPAP interfaces is available, including access to } \\
\text { humidification }\end{array}$ & \\
\hline Clear advice is offered to patients who drive about informing the DVLA & \\
\hline $\begin{array}{l}\text { Mandibular repositioning devices are available on the NHS to people } \\
\text { with mild to moderate OSA, with access to appropriately trained } \\
\text { personnel for fitting }\end{array}$ & \\
\hline Lifestyle advice is offered routinely as part of treatment for OSA & \\
\hline A local database provides up to date and accurate information & \\
\hline Follow-up after diagnosis and treatment is offered to all patients & \\
\hline Face to face follow up is available to those who need it (drivers etc.) & \\
\hline All patients have access to help and support by telephone & \\
\hline Medical devices and components are replaced as required & \\
\hline $\begin{array}{l}\text { The local model includes onward referral and partnership working } \\
\text { arrangements with relevant clinical services }\end{array}$ & \\
\hline $\begin{array}{l}\text { The local database provides up to date and accurate information } \\
\text { about treatment compliance and follow up }\end{array}$ & \\
\hline $\begin{array}{l}\text { Patient experience / satisfaction surveys are conducted every } \\
\text { year to inform service planning }\end{array}$ & \\
\hline
\end{tabular}




\section{Relevant resources:}

- National Institute for Health and Clinical Excellence (NICE) technology appraisal guidance 139 2008: Continuous positive airway pressure for the treatment of obstructive sleep apnoea/ hypopnoea syndrome

- IMPRESS 2009: Service Specification for Investigation and treatment of Obstructive Sleep Apnoea Syndrome

- ARTP 2010: Standards of care for sleep apnoea services (diagnostics)

- ARTP 2010: Standards of care for sleep apnoea services (CPAP)

- ARTP 2011: Standards of care for mandibular repositioning devices

- Mackay T 2010: Working towards the development of minimal standards for referral, investigation and treatment in Scotland (available on request from the BLF)

- Welsh Sleep Disordered Breathing Working Group 2010: Strategy Document for Sleep Disordered Breathing Services In Wales

- Northern Ireland OSAHS strategy group 2013: OSAHS Standards

- Primary Care Respiratory Society (PCRS-UK) 2013: Opinion sheet No. 53 - Sleep Apnoea

- BTS 2014: Position statement on driving and OSA

- OSA Partnership 2014: Four week wait campaign for vocational drivers

- OSA Map of Medicine - due to be published 2015

- North of England Specialist Commissioning Group (NW office) 2012: Sleep-Related Breathing Disorders (available on request from the BLF)

\section{BLF OSA online resources}

- OSA risk (mapping) in the UK

- Health Economics report

- Patient experience survey report

- Conference report

- Patient information

\section{Tool B: Planning local OSA services - OSA service calculator}

The OSA calculator tool is available on the BLF website: www.blf.org.uk/osa-calculator

You will be asked to type the name of your country and then to select your health area (Clinical Commissioning Groups (CCGs) in England, Health and Social Care Trusts (HSCTs) in Northern Ireland, NHS Health Boards (HBs) in Scotland and Local Health Boards (LHBs) in Wales).

You may need to wait while your report uploads.

You will see a report for your health area which you can download as a PDF file.

The report is illustrated with diagrams to explain key statistics and facts in your area. There is also the opportunity to generate reports about other areas if you work across more than one health area, or if you want to compare your report with a neighbouring area.

Finally you will be asked to give some feedback about the OSA toolkit, and to provide us with a contact email so that we might keep in touch to see how you get on.

N.B. The report should be used in conjunction with this booklet, which contains detailed information behind the figures given in the report. 


\section{Guidelines for interpreting the OSA calculator report}

You may need to wait while your report uploads.

Figure five overleaf illustrates what your report will look like.

\section{Estimated prevalence of moderate to severe OSA}

The first figure you will see is the estimated adult population with moderate to severe OSA in an average area of your population size. This number is calculated from ONS population figures and findings from the BLF OSA health economics report. ${ }^{67}$

\section{Weighted OSA risk estimate}

Next you will see the overall OSA risk band for your specific health area, compared with every other health area in the UK (Clinical Commissioning Groups (CCGs) in England, Health and Social Care Trusts (HSCTs) in Northern Ireland, NHS Health Boards (HBs) in Scotland and Local Health Boards (LHBs) in Wales).

The OSA risk is presented as a quintile band, where band five is the highest risk. It is based on actual local prevalence of risk factors for OSA which have been weighted and then an algorithm applied to calculate overall OSA risk. Being in a low risk area does NOT mean a low prevalence of OSA; it means that prevalence of risk factors in the area do not indicate an elevated prevalence of OSA. Being in a higher risk area indicates an increased risk of higher OSA prevalence.

You will also see the overall position for your area compared with the UK, where 1 is the lowest risk and 238 the highest.

Underneath the OSA risk diagram is a sentence to explain what this result could mean for your area.

\section{Risk factor prevalence}

Following this are the local risk quintiles and the ranks for the individual risk factors associated with
OSA: obesity, hypertension, diabetes, male gender, and age. These are based on actual prevalence data of these risk factors in your health area.

The overall OSA risk and the individual risk factor results were calculated as part of the BLF's OSA mapping project, published in $2013 .{ }^{68}$ See appendix for data sources and methodology used.

\section{NHS cost avoidance}

Following this is the estimated cost avoidances to the NHS of treating people with moderate to severe OSA with CPAP versus no treatment, and the estimated QALY change for treatment versus no treatment. These figures are based on the number of people in your area, and have been calculated from the BLF's health economics report. ${ }^{69}$

\section{Recommended staffing to run a full service} The updated recommended staffing levels for a population of your size are given, based on the most up to date findings from clinical practice, which are detailed in this booklet. These staffing levels are for a full service, i.e. the diagnosis, treatment and management of local people with OSA by a service that has been established for four years. Different models could be provided e.g.

- A new service could have fewer staff until the caseload builds

- Partnership arrangements between primary, secondary and tertiary care could be set up to spread the staffing resource across the health area

- Partnerships could be set up to outsource some elements of the service e.g. ongoing management and support

- The amount of medical time required may be less if there are trained and skilled technical/nursing staff available

\footnotetext{
${ }^{67}$ Office of Health Economics 2014: Obstructive sleep apnoea health economics report. Consulting report for the BLF

${ }^{68}$ Steier, J., Martin, A., Harris, J., Jarrold, I., Pugh, D. and Williams, A., 2014: Predicted relative prevalence estimates for obstructive sleep apnoea and the associated healthcare provision across the UK. Thorax, 69(4), pp. 390-392

${ }^{69}$ Office of Health Economics 2014: Obstructive sleep apnoea health economics report. Consulting report for the BLF
} 


\section{Estimated number of road accidents that could be prevented annually}

Finally an estimation of the local number of traffic accidents that could be prevented every year by treating all people with moderate to severe OSA is provided. This figure is based on a calculation in the BLF OSA health economics report, which cites the NICE costing template. ${ }^{70}$

The OSA report has been created to provide some information for local commissioning and service planning for OSA services, in conjunction with the audit checklist in this toolkit. It is important to stress that there will be local variation which should be considered, such as population density, location of hospitals, existing services and pathways, and identified need based on local knowledge of the population. Therefore we recommend that any service planning and commissioning involves involvement of all relevant local healthcare professionals and considers the needs of the local population.
Figure five:

Example of an OSA report for a named health area (in this case, a CCG in England)
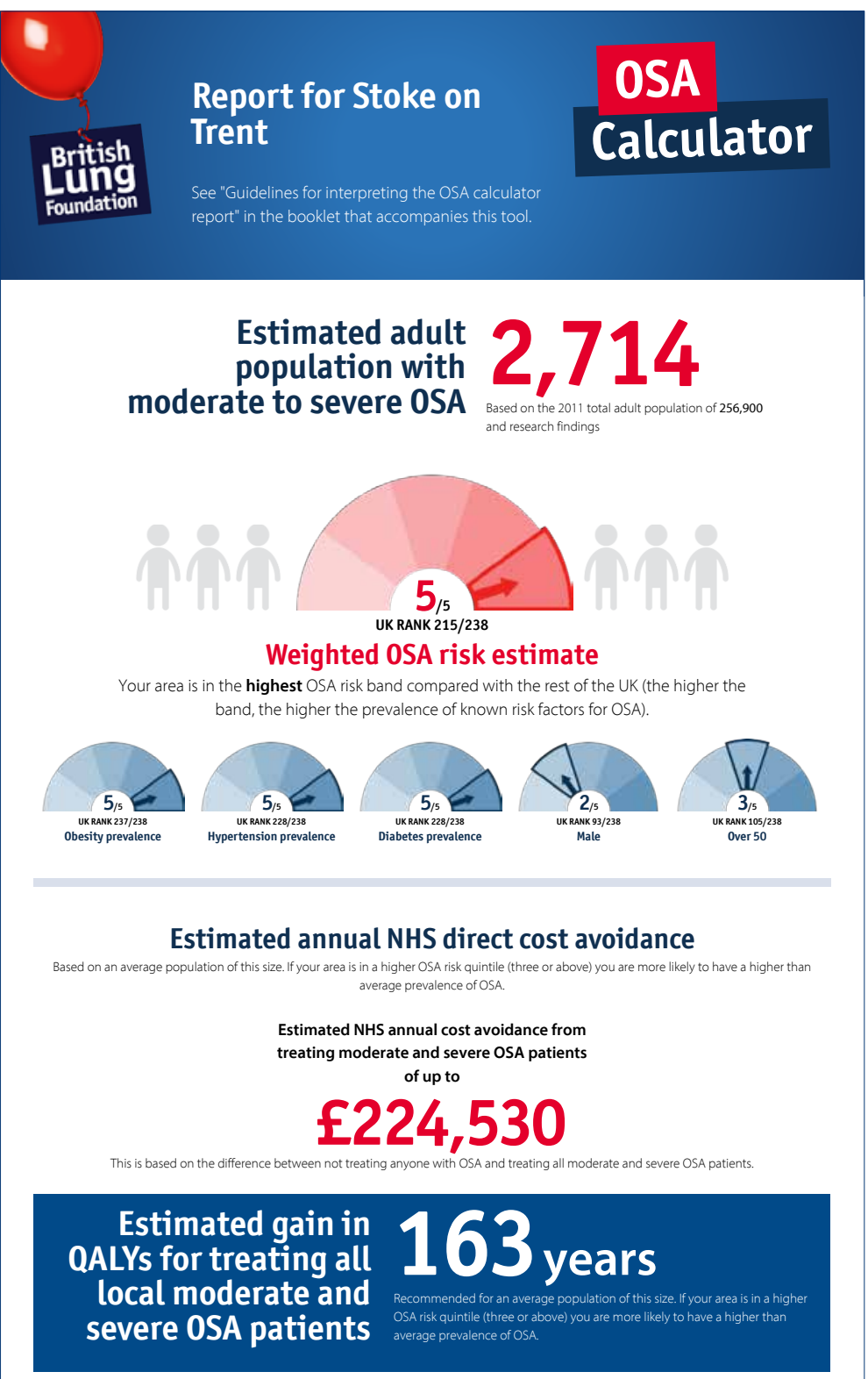

Recommended staffing to provide a diagnostic, treatment and support service

Recommended for an average popultion of this size and a service that has been established for approximately four years. If your area is in a higher Consultant programmed activities per week (DCC): 5.8 activities
Nursing/scientific/technical staff: 2.5 whole time equivalent
Secretarial/clerical staff: 1.4 whole time equivalent

Estimated number of road accidents that could be prevented annually by treating all moderate and severe OSA patients

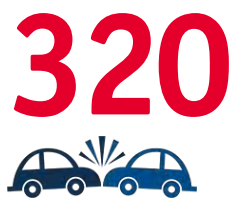




\section{Appendix}

\section{Referral protocol example}

2. Key performance indicators - outputs and outcomes

3. Patient survey example

4. Endorsements and acknowledgements

5. Data sources and methodology used to develop OSA risk map and calculator

\subsection{Referral protocol example}

Screenshot of part of online GP referral protocol developed by Dumfries and Galloway NHS for use by NHS Scotland health boards

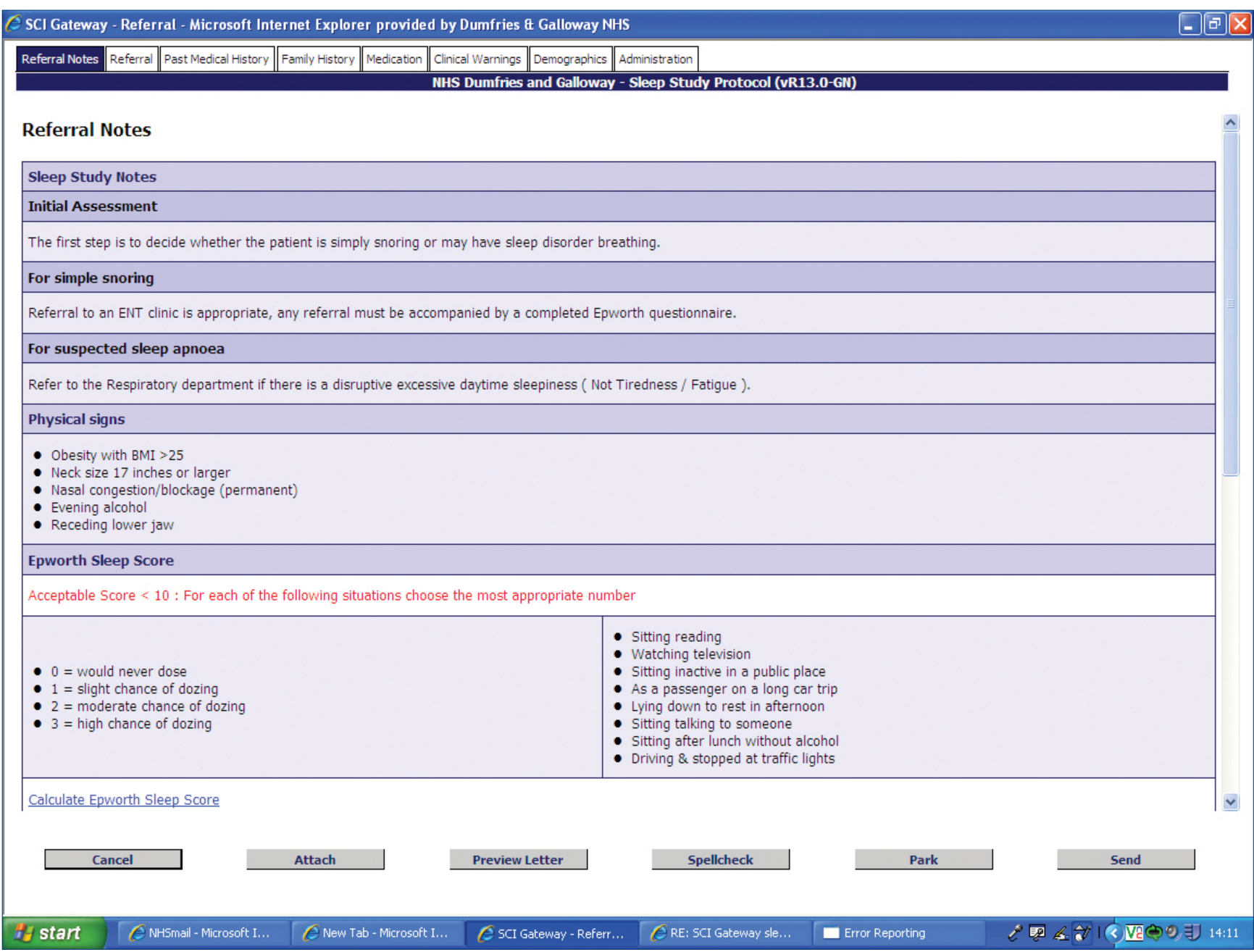


Example of a pathway, based on GP referral protocol developed by Dumfries and Galloway NHS

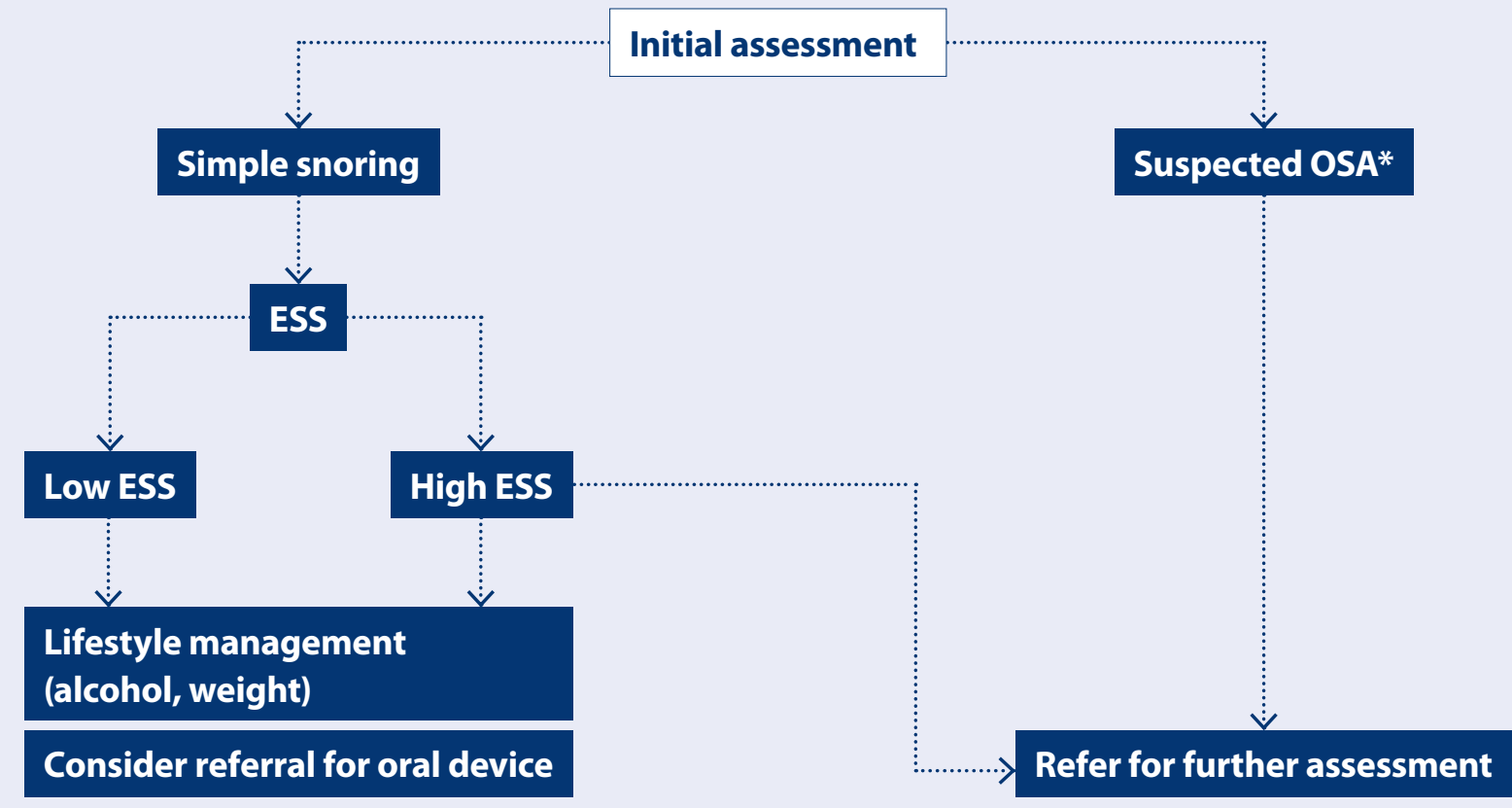

\section{*Suspected OSA:}

\section{Physical signs}

- Obesity with BMI over 25

- Neck size 17" or larger (men)

- Nasal congestion / blockage

- Evening alcohol

- Receding lower jaw

\section{ESS Score}

\section{Reason for referral}

- Provisional diagnosis / presenting complaint

- Urgency

- Temporary resident

\section{Specific questions}

- Witnessed apnoeas

- EDS

- Unrefreshing sleep

- Morning headache

- Loud snoring

- Restless sleep
- Disturbed sleep - frequent awakenings

- Choking sensation with abrupt awakening

- Other

ESS $=$ Epworth Sleepiness Scale $\mathrm{EDS}=$ Excessive daytime sleepiness

- Clinic information

- Pre-existing conditions

- Past procedures

- Family history

- Current medication

- Other medication

\section{Lifestyle risks}

- Weight / Height / BMI

- Smoking

- Alcohol

\section{Demographics}

- Patient number

- Name

- Address

- DOB

- Phone

- Email

- Sex

- Marital status

- Background data

- Driving status

7. Administration

- Date

- Vulnerable adult / child in need

- Referring GP details 


\subsection{Key performance indicators - outputs and outcomes}

\section{Suggested measurements}

\section{Annual outputs}

Number of:

- referrals to sleep clinics for assessment

- patients diagnosed with OSA (mild / moderate / severe)

- patients treated with CPAP

- patients treated with MRDs

- CPAP machines reclaimed

$\%$ of:

- patients with moderate or severe OSA who are CPAP compliant after one year (e.g. over 4 hours per night on at least 5 nights per week)

- patients satisfied with service (patient survey)

Changes in:

- Subjective assessment (e.g. Epworth score, quality of life)

- Objective assessment results (e.g. Apnoea Hypopnoea Index, oxygen saturation)
Waiting times:

- from referral to assessment

- from assessment to diagnosis

- from diagnosis to treatment

- for vocational drivers

Outcomes

- improved diagnosis rates

- reduced inappropriate referrals

- improved CPAP compliance

- improved quality of life (patient survey)

\section{Audit-based KPIs}

Specific KPIs can be devised based on results of the local audit (tool A in this toolkit).

These could include, for example:

- Regional service specification is developed

- Named clinical lead in each service

- GP education

- Development of new model / pathway

- Consistent use of resources e.g. for assessment, patient information 


\subsection{Patient survey}

Below is the patient experience survey used by the BLF in 2013. This can be used for follow-up surveys by clinics, health areas or regions.

\section{Section 1:}

\section{Questions about you}

\section{Q1. Age}

Under 18

18 or 19

$\square 20-29$

$\square 30-39$

$\square 40-49$

$\square 50-59$

$\square 60-69$

$\square 70-79$

$\square 0-89$

90 or over

Q2. Sex

$\square$ Male

$\square$ Female

Q3. Postcode (first 3 or 4 digits e.g. NW11)

Q4. Do you drive a motor vehicle (e.g. motor bike, car, lorry, bus)?

$\square$ Yes

$\square$ No

Q5. Do you hold a professional (e.g. HGV) driving license or drive for a living?

$\square$ Yes

$\square$ No
Q6. Do you have any of these conditions as well as OSA?

Please tick all that apply

$\square$ Angina

$\square$ Diabetes

$\square$ High blood pressure

$\square$ Previous strokes

$\square$ Previous heart attack

$\square$ Low thyroid

$\square$ Heartburn

$\square$ Weight problem (BMI of over 30)

$\square$ Other (please state)

Q7. Had you heard of OSA before you were diagnosed with it?

$\square$ Yes

$\square$ No

Q8. Who first realised that you had a problem?

Please tick one

$\square$ I realised myself

$\square$ My partner / spouse realised

$\square$ Another family member

$\square$ Friend

$\square$ Colleague

$\square$ Health care professional

$\square$ Other (please state)

Q9. How old were you when you first experienced symptoms of OSA? 
Q10. What symptoms did you have during the day?

Please tick all that apply

Sleepiness

Actually falling asleep

Waking feeling unrefreshed

Dry or sore throat

Morning headache

Poor concentration

Poor memory

Mood swings

Depression

Fell asleep driving

$\square$ Had road traffic accident caused by sleepiness

$\square$ Other (please state)

\section{Q11. What symptoms did you have} during the night?

Please tick all that apply

$\square$ Loud snoring

$\square$ Breathing pauses - stopping or struggling to breathe (witnessed by partner / friend)

Sudden or jerky body movements

$\square$ Restless sleep

$\square$ Frequent awakenings

Feelings of choking / gasping for breath

Needing the toilet often to pass urine

Heartburn

Other (please state)

\section{Q12. How long after first noticing your symptoms did you seek help?}

Please give time to the nearest year and month you can remember e.g. 4 years 3 months

\section{Q13. If you did not seek help as soon as you} noticed symptoms, why was this?

$\square$ I didn't believe there was anything wrong

I didn't want to admit I snored

$\square$ I was scared of losing my driving licence

I don't like going to the doctor

$\square$ I was scared of what it might be

Other (please state)

\section{Section 2:}

\section{Questions about the medical care you have received}

\section{Referral}

\section{Q14. Did you go to your GP about your} symptoms of OSA?

$\square$ Yes - please answer Q15 and Q16

$\square$ No - please go to Q17

\section{Q15. What happened?}

Please tick the one that is closest to your experience. I was...

Told not to worry, no further action was taken

$\square$ Given advice about stopping snoring, no further action was taken

$\square$ Given advice about losing weight, no further action was taken

$\square$ Referred to sleep clinic

Referred to ear, nose and throat clinic

Q16. How many times did you visit your GP about your OSA symptoms?

$\square 2$ 


\section{Q17. Did you try and treat your OSA} symptoms yourself?

Yes, tried to lose weight

Yes, tried to improve sleep habits

Yes, purchased a mouth guard from internet

Yes, purchased a CPAP machine from internet

Yes, other (please state)

$\square$ No

Q18. Which type of clinic were you referred to with your OSA symptoms?

ENT (Ear, nose and throat)

Respiratory (breathing / lung)

Specialist Sleep clinic

$\square$ Other (please state)

Q19. When were you first referred to a sleep clinic?

Please give date to the nearest month and year you can remember e.g. September 2010

Q20. When did you have your overnight sleep study?

Month and year

\section{Q21. When were you told of your} diagnosis of OSA?

Month and year

Q22. Who gave you your diagnosis?

$\square \mathrm{GP}$

$\square$ ENT physician

$\square$ Respiratory physician

$\square$ Nurse

Sleep technician or physiologist

$\square$ Other (please state)

Don't know

Q23. At the time of diagnosis, were you informed how severe your OSA was?

Yes - please go to Q24

$\square$ No - please go to Q25

$\square$ Don't remember - please go to Q25
Q24. Was it...

$\square$ Mild

$\square$ Moderate

$\square$ Severe

$\square$ Don't remember

Q25. How did you feel about your diagnosis?

Please tick the one that is closest to how you felt

Frightened

$\square$ Happy

$\square$ Relieved

$\square$ Resentful

$\square$ Surprised

$\square$ Worried

$\square$ Awkward

$\square \mathrm{Sad}$

$\square$ Hopeful

$\square$ Embarrassed

$\square$ Pleased

Q26. At the time of diagnosis, did you do a job that required you to drive regularly?

$\square$ Yes - please go to Q27

$\square$ No - please go to Q28

Q27. Did your employer understand your situation and reassure you about your continued employment?

$\square$ Yes

$\square$ No

$\square$ To some extent

$\square$ I didn't tell them

Q28. When you were diagnosed, were you given any information about OSA?

$\square$ Yes - please answer Q29 and Q30

$\square$ No - please go to Q31 


\section{Q29. Were you told about the following?}

Please tick all that apply

$\square$ Description of what happened during your sleep

$\square$ Causes of OSA

$\square$ Symptoms of OSA

$\square$ Impact OSA can have on health

$\square$ Informing the DVLA about your diagnosis of OSA

Treatments available for OSA

$\square$ What you could do to help yourself - e.g. stop smoking, lose weight, have regular routines, avoid alcohol and sleeping tablets

Q30. How were you given this information?

Please tick all that apply

$\square$ Leaflet, or other written information

$\square$ British Lung Foundation information

$\square$ Verbal information

Web-based

\section{Treatment}

Q31. What was the time gap between being diagnosed and receiving your first treatment?

$\square$ Same day

Next day

Within a week

Between one week and one month

$\square$ Between one and two months

$\square$ Between two and four months

Between four and six months

Between six months and a year

$\square$ Over a year

Q32. What treatment(s) did you have first?

Please tick all that apply

$\square$ Lifestyle advice (e.g. advised to lose weight, change sleep habits and routines)

CPAP machine

CPAP machine with humidifier

$\square$ Mouth guard (intra-oral device)

Surgery (please state what)

$\square$ Other (please state)
Q33. What difference did treatment make within the first month?

$\square$ It made my life worse

$\square$ No difference

$\square$ A bit of difference

$\square$ It helped quite a bit

$\square$ It helped a lot

$\square$ It changed my life for the better

Q34. What additional treatment(s) have you received since?

Please tick all that apply

$\square$ Lifestyle advice (e.g. advised to lose weight, change sleep habits and routines)

CPAP machine

CPAP machine with humidifier

Mouth guard (intra-oral device)

Surgery (please state what)

Other (please state)

None

Q35. What difference did this additional treatment make?

$\square$ It made my life worse

No difference

A bit of difference

$\square$ It helped quite a bit

$\square$ It helped a lot

$\square$ It changed my life for the better

Q36. What is the best treatment for you? (please state)

Q37. Are you still using this treatment?

$\square$ Yes

$\square$ No

Please only answer these questions (Q38 to Q46) if you have been given CPAP treatment. If you have never had CPAP, please go to Q47 


\section{Q38. Did you get used to CPAP?}

Yes, straight away

Yes, within one week

Yes, but it took a month

Yes, but it took 2-3 months

Yes, but it took 4-6 months

Yes, but it took me over 6 months

No, I could not get used to it

Q39. If you answered "No, I could not get used to it," what happened next?

Please tick all that apply

$\square$ I gave up and now have no treatment

I contacted my clinic but they couldn't really help

$\square$ I rang a helpline number / support group and this helped me

$\square$ I went back to my clinic and they helped me so I am now on CPAP (e.g. changed mask, changed settings)

I went back to my clinic and they gave me another treatment

\section{Q40. Did you receive a training session on how to} use your machine and how to fit your mask?

$\square$ Yes

$\square$ No

\section{Q41. When you went home with your CPAP, did} you feel confident about how to use it?

$\square$ Yes

$\square$ No

Q42. Did you have any problems with the mask, tubing or machine?

$\square$ Yes

$\square$ No

Q43. Were you given a helpline number or contact details of who to ring if you had problems?

$\square$ Yes

No
Q44. Are you still using CPAP now?

$\square$ Yes - please answer Q45 and Q46

$\square$ No - please go to Q47

Q45. How many days per week do you use your CPAP?

Q46. On average, how many hours do you use CPAP each night?

$\square 1$

$\square 2$

$\square 3$

$\square 4$

$\square 5$

$\square 6$

$\square 7$

$\square 8$

$\square 9$

Q47. What on-going care and support do you receive to help make sure you are managing your OSA?

Please tick the one that best applies

$\square$ I receive no support at all

$\square$ I can ring the clinic if I have any problems

$\square$ I receive a regular phone call to see how I am getting on

$\square$ I attend a drop-in to get my equipment checked and replaced

$\square$ I used to have check-ups but these stopped

$\square$ I attend a regular (e.g. annual) check-up appointment, which includes a discussion as well as getting my equipment serviced / replaced

Q48. Is your equipment regularly serviced / checked (e.g. once a year)?

$\square$ Yes

No 
Q49. How far do you have to travel to your sleep clinic?

$\square$ Less than 5 miles

$\square$ 5-10 miles

$\square$ 11-20 miles

$\square$ 12-30 miles

$\square$ Over 30 miles

Q50. Are you happy with the distance you have to travel?

$\square$ Yes

$\square$ No

\section{Section 3:}

Summary of your experience

Please give a summary of your experience of OSA before diagnosis and treatment:

Q51. I slept well at night

$\square$ Strongly agree

$\square$ Agree

$\square$ Neutral

$\square$ Disagree

Strongly disagree

Q52. I got up feeling refreshed

$\square$ Strongly agree

$\square$ Agree

$\square$ Neutral

$\square$ Disagree

$\square$ Strongly disagree

Q53. I felt sleepy in the day

$\square$ Strongly agree

$\square$ Agree

$\square$ Neutral

$\square$ Disagree

$\square$ Strongly disagree
Q54. I felt safe driving

$\square$ Strongly agree

$\square$ Agree

$\square$ Neutral

$\square$ Disagree

$\square$ Strongly disagree

Q55. My quality of life was excellent

$\square$ Strongly agree

$\square$ Agree

$\square$ Neutral

$\square$ Disagree

$\square$ Strongly disagree

Q56. My partner's quality of life was excellent

$\square$ Strongly agree

$\square$ Agree

$\square$ Neutral

$\square$ Disagree

$\square$ Strongly disagree

Q57. I felt happy

$\square$ Strongly agree

$\square$ Agree

$\square$ Neutral

Disagree

Strongly disagree

After diagnosis and treatment:

Q58. I slept well at night

$\square$ Strongly agree

$\square$ Agree

$\square$ Neutral

$\square$ Disagree

$\square$ Strongly disagree

Q59. I got up feeling refreshed

$\square$ Strongly agree

Agree

Neutral

Disagree

Strongly disagree 
Q60. I felt sleepy in the day

$\square$ Strongly agree

Agree

$\square$ Neutral

Disagree

Strongly disagree

Q61. I felt safe driving

$\square$ Strongly agree

Agree

$\square$ Neutral

Disagree

Strongly disagree

Q62. My quality of life was excellent

$\square$ Strongly agree

Agree

$\square$ Neutral

Disagree

Strongly disagree

Q63. My partner's quality of life was excellent

$\square$ Strongly agree

Agree

Neutral

$\square$ Disagree

Strongly disagree

Q64. I felt happy

Strongly agree

Agree

$\square$ Neutral

Disagree

Strongly disagree

\section{Section 4: What you would like} services to look like

Where do you think future OSA services should be located?

Q65. Assessment - tests to find out what is causing your symptoms

$\square$ In a specialist sleep centre in your region (e.g. county or city)

$\square$ At your local hospital

At your GP surgery

At another GP surgery, still close

$\square$ to your home, offering a service

$\square$ to local people

By telephone, or video call, to a sleep centre

Q66. Diagnosis - being told you have OSA

$\square$ In a specialist sleep centre in your region (e.g. county or city)

$\square$ At your local hospital

$\square$ At your GP surgery

$\square$ At another GP surgery, still close to your home, offering a service to local people

By telephone, or video call, to a sleep centre

Q67. Treatment - being given your treatment and shown how to use it

$\square$ In a specialist sleep centre in your region (e.g. county or city)

$\square$ At your local hospital

$\square$ At your GP surgery

$\square$ At another GP surgery, still close to your home, offering a service to local people

$\square$ By telephone, or video call, to a sleep centre 
Q68. On-going support - to check your equipment and make sure you are getting on ok

$\square$ In a specialist sleep centre in your region (e.g. county or city)

$\square$ At your local hospital

$\square$ At your GP surgery

$\square$ At another GP surgery, still close to your home, offering a service to local people

$\square$ By telephone, or video call, to a sleep centre

Q69. If you were also due to visit another clinic, such as a weight clinic, a diabetes clinic, or a blood pressure clinic, would you prefer to go to one general clinic for your different conditions, or to visit specialist clinics separately?

$\square$ I would prefer to go to one general clinic

$\square$ I would prefer to go to different specialist clinics for each condition 


\subsection{Endorsements and acknowledgements}

\section{Endorsements and supporting statements}
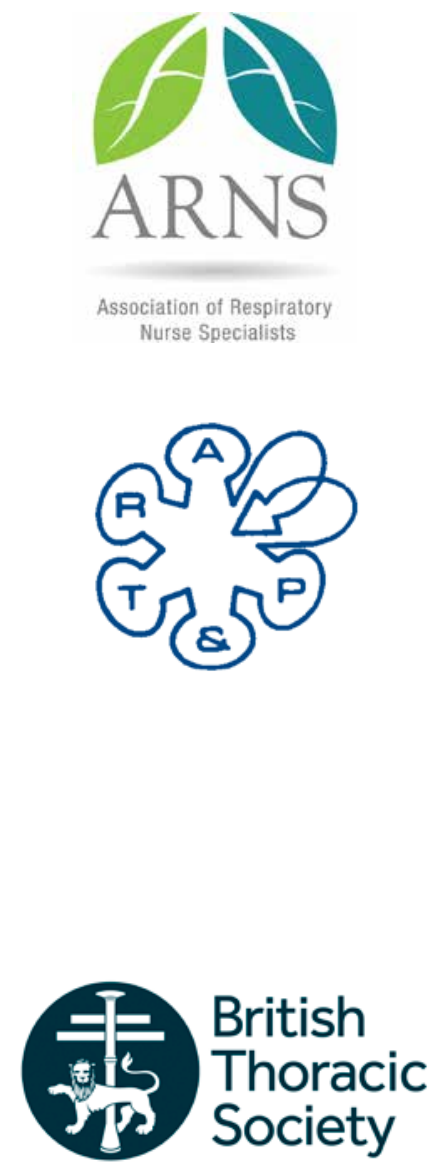

\section{Association of Respiratory Nurse Specialists (ARNS)}

Nurses in particular play a major role in advising and supporting commissioners wanting to set up and run OSA services. This brilliantly comprehensive tool pack will ensure that nurses and other healthcare professionals are fully supported so that they can easily build successful cases for adequate service provision and service plans. Rebecca Sherrington, Chair ARNS

\section{Association of Respiratory Technology and Physiology (ARTP)}

In the wake of the obesity epidemic, obstructive sleep apnoea could potentially swamp our healthcare system, but this BLF OSA document has taken the best advice to ameliorate the likely impact of OSA on the UK healthcare systems. By screening for those at highest risk, getting the right diagnosis, giving them the right treatment and providing the best advice, our patients will receive the service they deserve - the best. This will be another success story for the NHS which will remain the envy of other healthcare systems.

\section{Dr Brendan G Cooper, President, ARTP}

\section{British Thoracic Society (BTS)}

BTS welcomes the production of this toolkit which will be valuable for commissioners and providers of services and, ultimately of benefit to people with OSA. While local variations continues to exist, both in provision of services and the resources available to treat the condition, the toolkit provides valuable practical assistance and will also help to raise the profile of this condition and the need to provide early and appropriate treatment.

\section{Dr Martin Allen, Honorary Secretary, BTS}

\section{British Sleep Society (BSS)}

Adequate and undisturbed sleep is essential for the health of the individual,

British Sleep Society and for the health of the society of which we are all part. Obstructive Sleep Apnoea is amongst the commonest of sleep disturbances, directly impacting on such health, and yet is sorely under diagnosed with predictable and expensive knock-on effects on cardiovascular, metabolic and mental health. The tool kit presented here should go some way to address this, and as such is a welcome resource.

Professor Adrian Williams, BSS committee 


\section{OSA Partnership Group}

The OSA Partnership Group is running a campaign for the fast tracking of treatment for vocational drivers with OSA to ensure these drivers have the confidence to present for diagnosis and treatment. Our campaign has highlighted that CCGs and individual sleep clinics take different approaches to OSA treatment and its prioritization. Having reviewed the BLF toolkit we believe that it will provide CCGs with valuable information that will help in the planning and commissioning of treatment, support more consistency of approach, and hopefully encourage the fast tracking we are seeking. This will have a positive impact on patients and their families, as well as supporting a reduction in the number of related road traffic accidents. We are very pleased to endorse this initiative which should encourage greater emphasis on OSA treatment.

\section{Gillian Gibbons, Chair, OSA partnership}

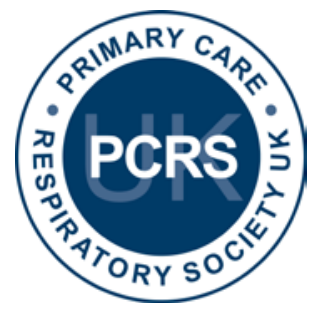

\section{Primary Care Respiratory Society UK (PCRS-UK)}

Primary Care Respiratory Society UK is pleased to see this useful toolkit on commissioning and planning local services for obstructive sleep apnoea. The tools will help both commissioners and clinicians to audit existing services, and to plan future services for people with sleep apnoea, which is a serious and under-recognised health issue.

\section{Acknowledgements}

\section{The BLF would like to thank:}

1. ResMed for funding the BLF OSA toolkit and health economics report. ResMed, a medical device company, has had no influence over the contents of this toolkit

2. Philips Respironics, the founding partner of the BLF OSA project

\section{Professor John Stradling}

4. OSA healthcare professionals who have supported our work tirelessly and without whom we could not have produced this toolkit. In particular, thanks to Drs Martin Allen, Brendan Cooper, John O'Reilly and Eric Livingston

5. Colleagues who piloted the draft toolkit across the UK, especially: Tahmina Siddiqui, Alex Perkins, David Sword, Jacqui Meggary, Steve Eastwood
7. Association of Respiratory Technology and Physiology

8. British Thoracic Society

9. British Sleep Society

10. OSA Partnership Group

11. Primary Care Respiratory Society UK

12. The Office of Health Economics

13. Patient Perspective

14. Lovell Johns

15. Headscape

6. Association of Respiratory Nurse Specialists 


\subsection{Data sources and methodology used to develop OSA risk map and calculator}

Extensive consultation with the BLF OSA advisory group led to the identification of a set of risk factors for OSA. These were:

- Age - OSA is more prevalent in older people (Young et al, 2002)

- Hypertension prevalence - hypertension can be caused by OSA (Levy et al 2011)

- Obesity prevalence - Obesity is present in about 40\% of OSA patients (Levy et al 2011)

- Type 2 diabetes prevalence - recent reports have indicated that the majority of patients with type 2 diabetes have OSA (Levy et al 2011)

- Sex - OSA is more common in men than women (Young et al 1993)

Based on their knowledge of OSA research, advisory group members ranked the 5 risk factors as follows:

- obesity (5)

- diabetes (4)

- age (3)

- hypertension (2)

- $\operatorname{sex}(1)$

Lovell Johns then applied a calculation to the risk factors based on advice from members of the OSA Advisory Group:

a) There are 5 risk factors, ranked strongest (5) to weakest (1)

b) For each risk factor, the areas (e.g. constituencies) were ranked based on the risk factor prevalence, and then split the areas into 5 risk bands (quintiles), with a fifth of the areas in each band. The fifth of areas with the highest prevalence score 5, down to the lowest prevalence band scoring 1.

For the top ranked factor, obesity, the risk band score (1-5) was multiplied by 5 , giving weighted risk scores of 25 (for highest prevalence areas), 20, 15, 10 or 5 (lowest prevalence areas).
The same was done for the other risk factors, down to the lowest ranked factor, sex, where the risk score was multiplied by 1 , giving weighted risk scores of 5 (highest prevalence areas), 4, 3, 2 or 1 (lowest prevalence areas).

The overall risk score was made by summing the 5 scores.

This means that areas in the highest risk band for all risk factors would always be at the top of the overall risk list, followed by areas in the highest risk band for the strongest 4 factors and in the second highest risk band for the weakest factor, and so on.

Lovell Johns extrapolated raw data from various sources to produce a series of risk factor maps. These data sources included:

\section{OSA Risk Layers - spatial datasets}

- Office for National Statistics (with Ordnance Survey), GB health area vector boundaries

- NHS Commissioning Board website, table of LSOAs making up each provisional CCG

- Ordnance Survey, Boundary Line - for GB Constituency boundaries

- Land and Property Services Northern Ireland, 1:50,000 scale Westminster Parliamentary Constituency vector boundaries

- NHS Connecting for Health website, epraccur Table of England and Wales GP codes and postcodes

- Ordnance Survey Codepoint (with Royal Mail) for geolocating GP surgeries by postcode

OSA Risk Layers - demographics

- Office for National Statistics, Mid-2010 Population Estimates for Parliamentary Constituencies in England and Wales by Quinary Age and Sex

- Office for National Statistics, Mid-2010 Population Estimates for LSOAs by single year of age, Males \& Females - for CCGs 
- Office for National Statistics, Mid-2010 Population Estimates: Quinary age groups for health areas in the United Kingdom - for Welsh local health boards

- Northern Ireland Statistics and Research Agency, HSCT Population by 5yr age group (and sex) 1991-2010

- Northern Ireland Statistics and Research Agency, Mid-Year population estimates (2010) for 18 Westminster Parliamentary Constituencies / NI Assembly Areas in Northern Ireland

- National Records of Scotland, Estimated population by sex, single year of age and Westminster Parliamentary Constituency area mid-2001 to mid-2010

- National Records of Scotland, Estimated population by sex, single year of age and Scottish Parliamentary Constituency area mid-2001 to mid-2011

- National Records of Scotland, Estimated population by sex, five year age group and administrative area: 30 June 2010 for NHS board demographics

\section{OSA Risk Layers - disease prevalence data}

- Health and Social Care Information Centre, Quality and Outcomes Framework Apr 2010 Mar 2011, England: Numbers on QOF disease registers and raw prevalence rates by Practice

- Department of Health, Social Services \& Public Safety (Northern Ireland), Quality and Outcomes Framework (QOF) Disease Prevalence Data 2011 by geographies incl. WPC, HSCT

- ISD Scotland, Quality \& Outcomes Framework (QOF) for April 2010 - March 2011, Scotland: Reported prevalence/register information by practice, by health board

- Welsh Government, Information from the General Medical Services Quality and Outcomes Framework Statistics for Wales, 2010-11

Other

- NHS Right Care, Atlas of Variation for Respiratory Disease 2012 - for rates of sleep studies by PCT

Background Maps

- Ordnance Survey Miniscale raster

- Ordnance Survey 1:250,000 scale raster background map on London regional map 

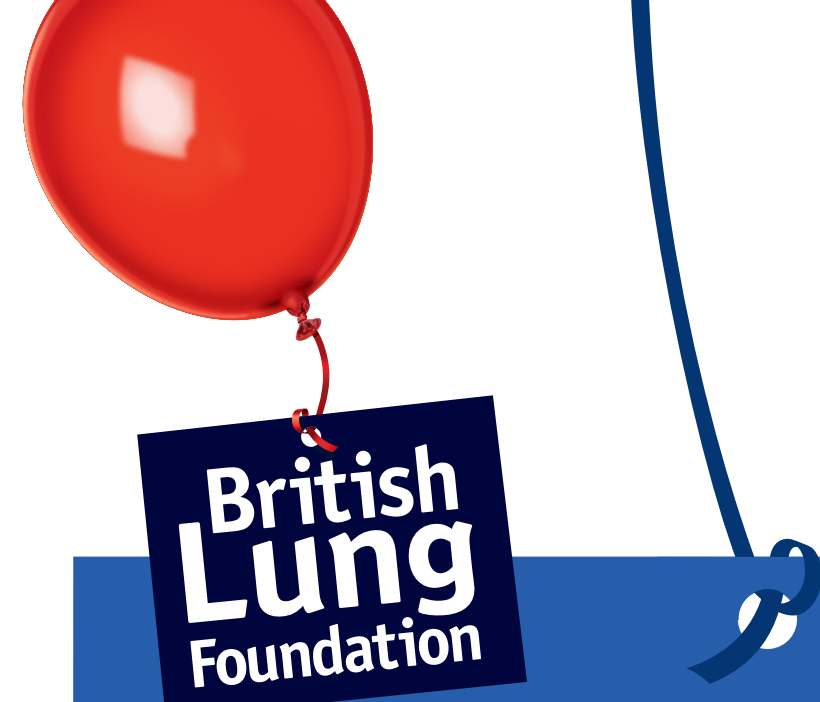

One person in five in the UK is affected by lung disease. Millions more are at risk.

We are the UK's lung charity and we are here for every one of them, whatever their condition.

Lung disease can be frightening and debilitating. We offer hope and support at every step so that no one has to face it alone.

We promote greater understanding of lung disease and we campaign for positive change in the nation's lung health.

We fund vital research, so that new treatments and cures can help save lives.

We are the British Lung Foundation. Leading the fight against lung disease.

's 